\title{
Neuron-specific PERK inactivation exacerbates neurodegeneration during experimental autoimmune encephalomyelitis
}

\author{
Sarrabeth Stone, ${ }^{1,2}$ Yuan Yue, ${ }^{1,2}$ Milos Stanojlovic, ${ }^{1,2}$ Shuangchan Wu, ${ }^{1,2}$ Gerard Karsenty, ${ }^{3}$ \\ and Wensheng Lin ${ }^{1,2}$ \\ 'Department of Neuroscience and 'Institute for Translational Neuroscience, University of Minnesota, Minneapolis, \\ Minnesota, USA. ${ }^{3}$ Department of Genetics and Development, Columbia University College of Physicians and Surgeons, \\ New York, New York, USA.
}

\begin{abstract}
Multiple sclerosis (MS) and its animal model, experimental autoimmune encephalomyelitis (EAE), are chronic inflammatory demyelinating and neurodegenerative diseases of the CNS. Although neurodegeneration is the major contributor to chronic disability in MS, mechanisms governing the viability of axons and neurons in MS and EAE remain elusive. Data indicate that activation of pancreatic endoplasmic reticulum kinase (PERK) influences, positively or negatively, neuron and axon viability in various neurodegenerative diseases through induction of ATF4. In this study, we demonstrate that the PERK pathway was activated in neurons during EAE. We found that neuron-specific PERK inactivation impaired EAE resolution and exacerbated EAE-induced axon degeneration, neuron loss, and demyelination. Surprisingly, neuron-specific ATF4 inactivation did not alter EAE disease course or EAE-induced axon degeneration, neuron loss, and demyelination. These results suggest that PERK activation in neurons protects axons and neurons against inflammation in MS and EAE through ATF4-independent mechanisms.
\end{abstract}

Authorship note: SS and YY contributed equally to this work.

Conflict of interest: The authors have declared that no conflict of interest exists.

License: Copyright 2019, American Society for Clinical Investigation.

Submitted: August 14, 2018 Accepted: December 5, 2018 Published: January 24, 2019

\section{Reference information:} JCI Insight. 2019;4(2):e124232. https://doi.org/10.1172/jici. insight.124232.

\section{Introduction}

Multiple sclerosis (MS) is a chronic inflammatory disease of the CNS $(1,2)$. Although myelin and oligodendrocytes are the primary target of inflammatory attacks, it is the neurodegeneration, particularly axon degeneration, which leads to long-term disability in MS patients (2-4). It is known that axon degeneration occurs early in MS lesions $(5,6)$. Significant neuron loss is also observed in the CNS gray matter in MS patients $(7,8)$. Moreover, magnetic resonance image studies showed that brain atrophy occurs very early in MS patients and that progressive brain atrophy correlates well with disability $(9,10)$. The MS animal model experimental autoimmune encephalomyelitis (EAE) recapitulates many of the clinical, pathological, and immunological features of MS, including neurodegeneration (11-13). The current predominant view is that inflammation is ultimately responsible for axon degeneration and neuron loss in MS and EAE; however, the molecular mechanisms of neurodegeneration in these diseases remain ambiguous (14-16). One of the major challenges in MS research is to understand the mechanisms governing the viability of axons and neurons and to develop therapeutic strategies that prevent neurodegeneration.

Endoplasmic reticulum (ER) stress activates the unfolded protein response (UPR), an adaptive response that is coordinated by 3 ER-resident transmembrane proteins: pancreatic ER kinase (PERK), inositol requiring enzyme 1 (IRE1), and activating transcription factor $6 \alpha$ (ATF6 $\alpha$ ) (17-19). PERK activation triggers an adaptive program known as the integrated stress response (ISR) by phosphorylating eukaryotic translation initiation factor $2 \alpha(\operatorname{IF} 2 \alpha)(17-19)$. eIF $2 \alpha$ phosphorylation (p-eIF2 $\alpha$ ) results in inhibition of global protein translation with a selective increase in the translation of ATF4. ATF4 stimulates the expression of numerous cytoprotective genes and also the proapoptotic transcription factor CAATT enhancer-binding protein homologous protein (CHOP). CHOP induction also leads to downregulation of the ISR by enhancing the expression of growth arrest and DNA damage 34 (GADD34), which functions with protein phosphatase 1 (PP1) to dephosphorylate p-eIF2 $\alpha$, forming 
a tight negative feedback loop. Conversely, persistent activation of the ISR can result in cell apoptosis through inhibition of protein translation and/or induction of CHOP $(20,21)$. ER stress is an important feature of inflammatory diseases, including MS and EAE (22-24). The PERK-mediated ISR is activated in several cell types in MS and EAE, including oligodendrocytes, neurons, T cells, astrocytes, and microglia/macrophages (25-27). Data from our lab and other groups have demonstrated that the PERK-mediated ISR is a major player in regulating oligodendrocyte viability during EAE, protecting oligodendrocytes and myelin against inflammation (28-32).

While a large number of studies have shown that the PERK-mediated ISR plays a critical role in various neurodegenerative diseases, these studies are at times contradictory. Some studies showed that the ISR promotes neuron and axon survival in neurodegenerative diseases; however, other studies showed opposite results (33-35). Nevertheless, the role that the PERK-mediated ISR in neurons plays in axon degeneration and neuron loss in MS and EAE remains unknown. This study sought to fill this knowledge gap. Herein, we demonstrated activation of the PERK-mediated ISR in neurons during EAE. We found that neuron-specific PERK inactivation did not affect EAE initiation but impaired EAE recovery, which was associated with increased axon degeneration, neuron loss, and demyelination in the CNS. Surprisingly, we found that neuron-specific ATF4 inactivation did not alter EAE disease severity or EAE-induced neurodegeneration. These findings imply the neuroprotective effects of PERK activation in neurons in MS and EAE via ATF4-independent mechanisms.

\section{Results}

The PERK-mediated ISR was activated in neurons during EAE. Evidence suggests activation of the PERK-mediated ISR in neurons in the CNS of MS patients $(26,36)$. We have used myelin oligodendrocyte glycoprotein (MOG) peptide 35-55-induced EAE in C57BL/6J mice (MOG-EAE model) to study the pathogenesis of MS and EAE for many years (28-31, 37-39). When young adult female C57BL/6J mice are immunized with MOG 35-55 peptide, the mice display neurological signs of disease starting around postimmunization day (PID) 12, reach the peak of disease around PID 19-26, and then gradually recover from EAE over time. Our previous studies demonstrated axon degeneration in the lumbar spinal cord and neuron loss in the CNS gray matter in the MOG-EAE model at the peak of disease $(29,30,39)$. Therefore, we sought to determine activation of the PERK-mediated ISR in neurons in the MOG-EAE model.

Although we have worked in the ER stress field for many years, we could not find any reliable antiPERK antibody or anti-phosphorylated PERK antibody for Western blot or IHC. Thus, we used Western blot analysis for ATF4 and CHOP as well as IHC for p-eIF2 $\alpha$ and CHOP to gauge the activity of the PERK-mediated ISR in neurons. Western blot analysis showed that the levels of ATF4 and CHOP were significantly increased in the brains of wild-type mice with EAE at the peak of disease, compared with that in naive wild-type mice (Figure 1, A and B). Western blot analysis also showed that the level of cleaved ATF6 $\alpha$ was significantly elevated in the brains of wild-type mice with EAE compared with that in naive wild-type mice, suggesting activation of the ATF $6 \alpha$ branch of the UPR in the brains of mice with EAE (Figure 1, A and B). p-eIF2 $\alpha$ and NeuN double immunostaining showed that the immunoreactivity of p-eIF2 $\alpha$ was barely detectable in neurons in the CNS of naive wild-type mice. Interestingly, the level of p-eIF2 $\alpha$ was markedly increased in the majority of neurons in the CNS of wild-type mice with EAE at the peak of disease (Figure 1C). CHOP and NeuN double immunostaining showed that the immunoreactivity of CHOP was barely detectable in neurons in the CNS of naive wild-type mice. Importantly, the level of CHOP was markedly increased in the majority of neurons in the CNS of wild-type mice with EAE at the peak of disease (Figure 1D). Moreover, nuclear translocation of CHOP was observed in a number of neurons in the CNS of wild-type mice with EAE (Figure 1D), which strongly indicates activation of the PERK-mediated ISR in these cells (17-19). Taken together, these data suggest activation of the UPR, including the ATF6 $\alpha$ branch and PERK-mediated ISR, in neurons in the CNS during EAE.

PERK was not required for neuron or axon survival in adult mice. Recently, a case study described early signs of neurodegeneration in a child who carries a PERK mutation (40), suggesting the potential role of PERK in neurons under physiological conditions. Thus, we determined the effects of PERK inactivation on the viability of neurons and axons in naive adult mice using a mouse model that allows for controllable inactivation of PERK specifically in neurons. PERK ${ }^{\text {loxP }}$ mice that possess loxP sites flanking exons 3-5 of the Perk gene (41) were crossed with Thy1/CreER ${ }^{T 2}$ mice that express CreER ${ }^{\mathrm{T} 2}$ selectively and ubiquitously in neurons in the CNS (42), and the resulting progeny were further crossed with $P E R K^{\text {loxP }}$ mice to obtain $P E R K$ - 

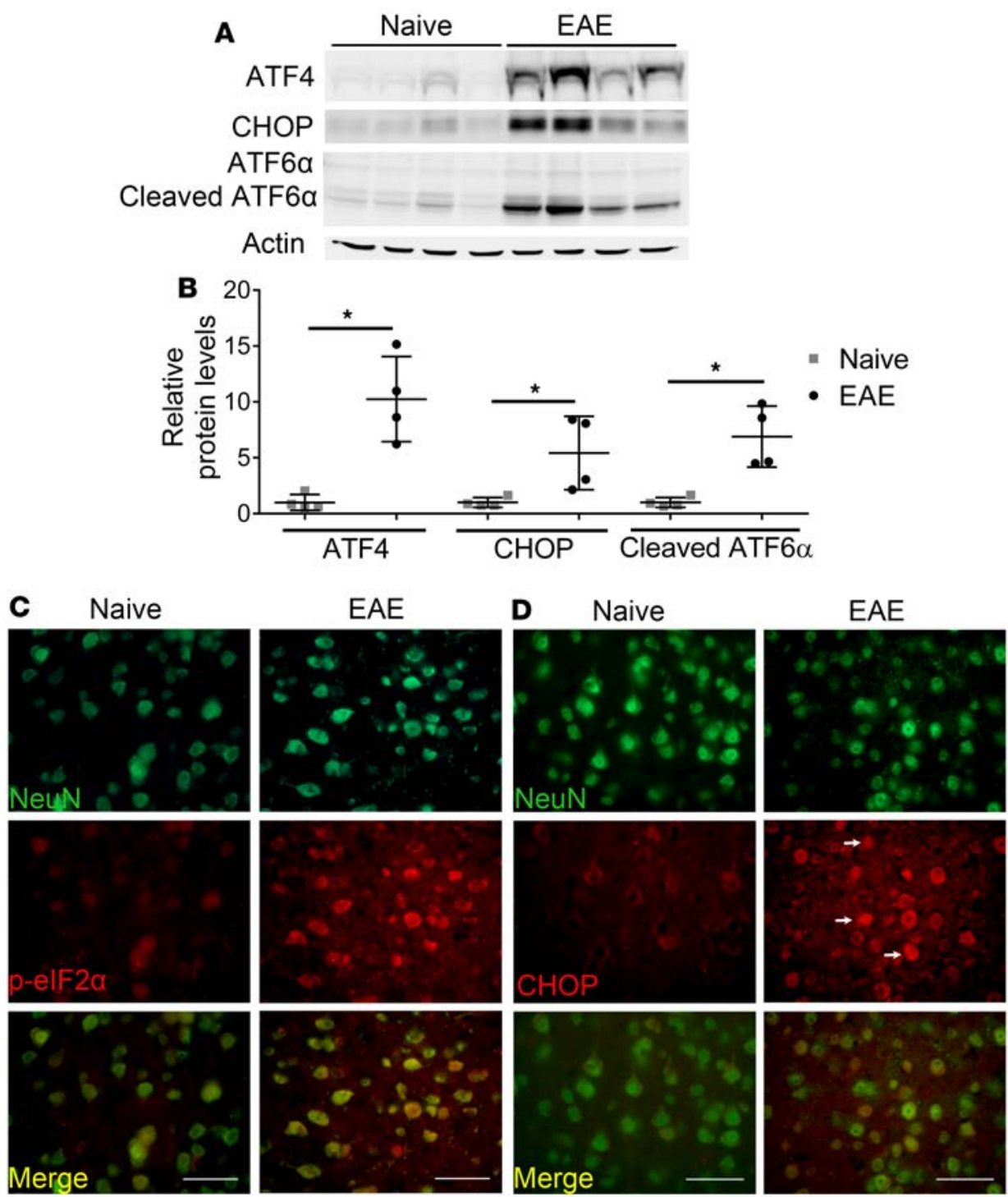

Figure 1. The PERK-mediated ISR was activated in neurons in the MOG-EAE model. (A and B) Western blot analysis shows that the levels of ATF4, CHOP, and cleaved ATF6 $\alpha$ were significantly increased in the brains of wild-type mice with EAE at the peak of disease (PID 19) compared with those in naive wild-type mice. (C) NeuN and p-elF2 $\alpha$ double immunostaining shows that the level of p-elF2 $\alpha$ was markedly increased in neurons in the layer $V$ of the primary motor cortices of wild-type mice with EAE at the peak of disease (PID 19) compared with that in naive wild-type mice. (D) NeuN and CHOP double immunostaining shows the elevated level of CHOP and nuclear translocation of CHOP (arrows) in neurons in the layer $V$ of the primary motor cortices of wild-type mice with EAE at the peak of disease (PID 19) compared with that in naive wild-type mice. Scale bars: $50 \mu \mathrm{m} . n=4$ animals. Error bars represent SD. Statistical analyses were done with a 2-tailed $t$ test, ${ }^{*} P<0.05$.

loxP/loxP; Thy1/CreER ${ }^{T 2}$ mice, PERK ${ }^{\text {loxP/loxP }}$ mice, and Thy1/CreER $R^{T 2}$ mice. Seven-week-old PERK ${ }^{\text {loxP/loxP }}$; Thy1/ $C r e E R^{T 2}$ mice were given i.p. injections of tamoxifen or vehicle daily for 8 consecutive days. The tamoxifen-treated mice were phenotypically normal and indistinguishable from the vehicle-treated mice. CNS tissues, other tissues, and purified splenic T cells were prepared from these mice 8 weeks after tamoxifen treatment. PCR analysis of genomic DNA revealed the deletion of exons 3-5 of the Perk gene selectively in the CNS of PERK $K_{\text {loxP/loxP }}$; Thy $1 / C r e E R^{T 2}$ mice treated with tamoxifen (PERK-nKO mice) but not in other organs or purified $\mathrm{T}$ cells of PERK-nKO mice or in any organs or T cells of PERK loxP/loxP ; Thy1/CreER ${ }^{T 2}$ mice treated with vehicle (control mice) (Figure 2A). Moreover, real-time PCR analysis showed that the level of PERK mRNA was significantly reduced in the cortices and hippocampi of PERK-nKO mice compared with that in control mice (Figure 2B). H\&E staining showed that PERK-nKO mice did not display any gross structural abnormalities in the CNS. Moreover, NeuN IHC revealed that PERK-nKO mice had a 


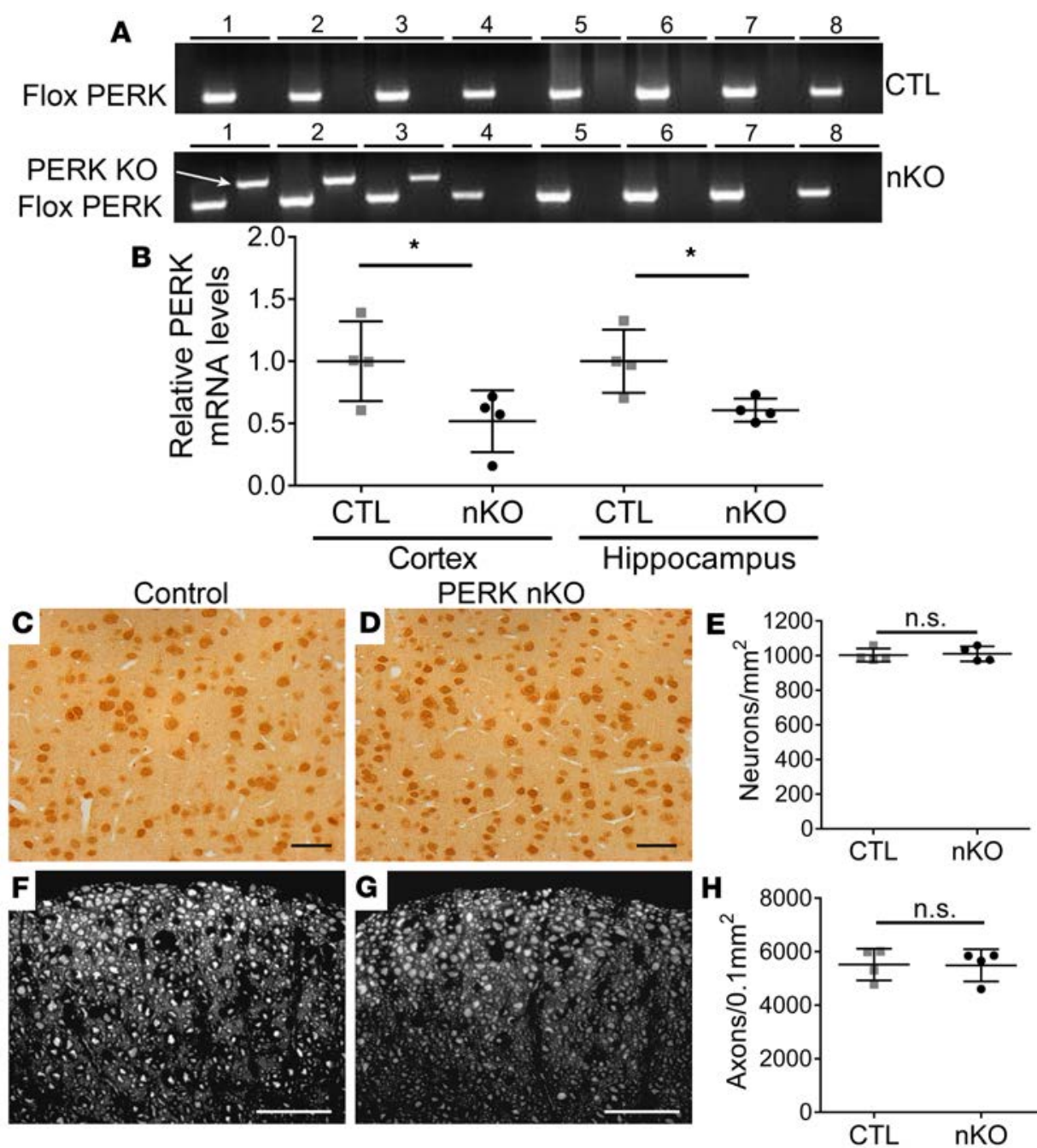

Figure 2. Neuron-specific PERK inactivation did not alter the viability of neurons or axons under physiological conditions. (A) PCR analysis using genomic DNA shows the floxed Perk allele was present in all tissues in PERK-nKO mice (nKO) and control mice (CTL), but the Perk-KO allele was only present in the cerebral cortex, cerebellum, and spinal cords of PERK-nKO mice. Numbering above lanes is as follows: 1, cerebral cortex; 2, cerebellum; 3, spinal cord; 4, optic nerve; 5, sciatic nerve; 6, spleen; 7, lymph node; 8, T cells. (B) Real-time PCR shows that the level of PERK mRNA was significantly decreased in the cortices and hippocampi of PERK-nKO mice compared with control mice. (C-E) NeuN IHC shows that neuron-specific PERK inactivation did not significantly change the number of neurons in layer $V$ of the primary motor cortices of adult mice. (F-H) SMI31 IHC shows that neuron-specific PERK inactivation did not significantly change the number of axons in the lumbar spinal cords of adult mice. Scale bars: $50 \mu \mathrm{m} . n=4$ animals. Error bars represent SD. Statistical analyses were done with a 2-tailed $t$ test, $* P<0.05$.

similar number of neurons in the layer $\mathrm{V}$ of the primary motor cortex compared with control mice (Figure 2, C-E). Similarly, phosphorylated neurofilament-H (SMI31) IHC revealed that the number of axons in the lumbar spinal cord was not compromised in PERK-nKO mice compared with that in control mice (Figure 2, F-H). These data suggest that PERK is dispensable for neuron and axon survival in naive adult mice.

Neuron-specific PERK inactivation exacerbated EAE-induced axon degeneration and neuron loss. To determine the effects of PERK inactivation in neurons in EAE, 7-week-old female $P E R K^{\text {loxPlloxP }}$; Thy $1 / C_{r e E R^{T 2}}$ mice were given i.p. injections of tamoxifen daily for 8 consecutive days, and then these mice were immunized with MOG 35-55 peptide to induce EAE at the age of 9 weeks (PERK-nKO mice). Control EAE mice included age-matched PERK ${ }^{\text {toxPl/oxP}}$; Thy $1 / C r e E R^{T 2}$ mice treated with vehicle, $P E R K^{\text {loxPlloxP }}$ mice treated with tamoxifen, and Thy $1 / \mathrm{CreER}{ }^{T 2}$ mice treated with tamoxifen. As expected, control EAE mice displayed a typical EAE disease course, with disease onset around PID 12, reaching the peak of disease around PID 19-26, and remitting later in the disease course (Figure 3A). Although disease onset and the time at which the peak of disease was reached in PERK-nKO mice with EAE were comparable to those of control EAE mice, these PERK-nKO 


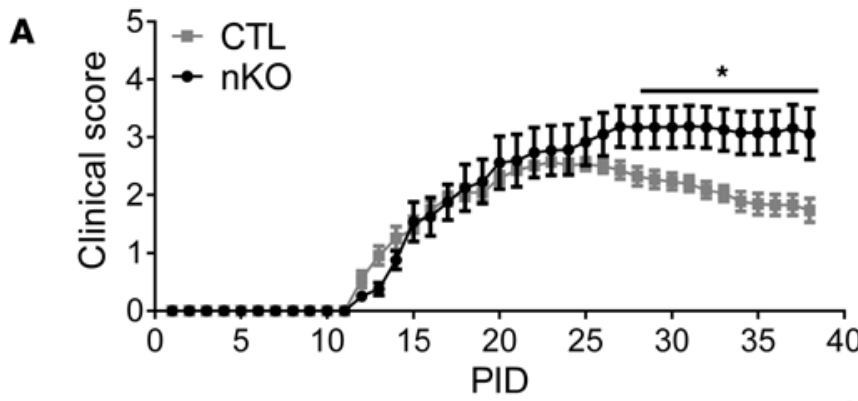

B

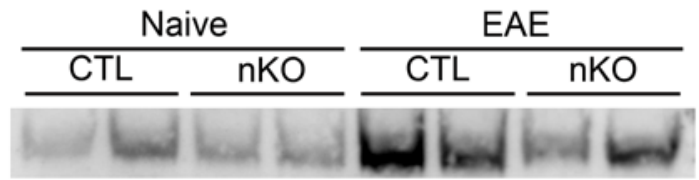

CHOP

$\beta$-Actin

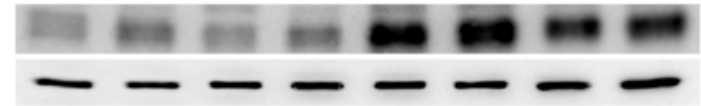

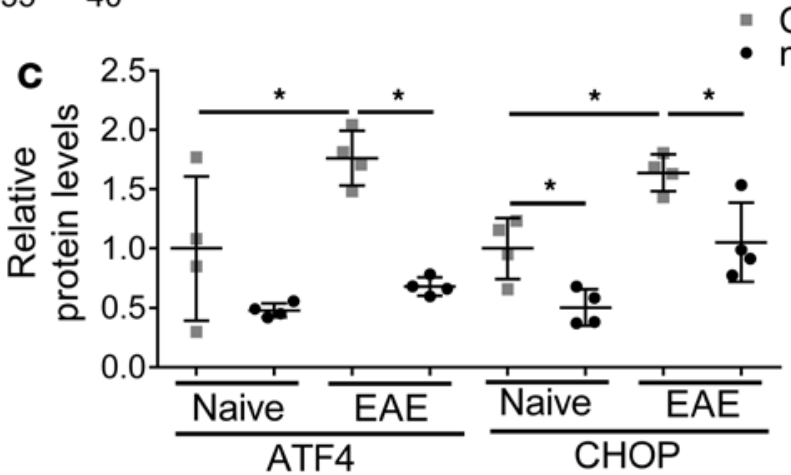

D

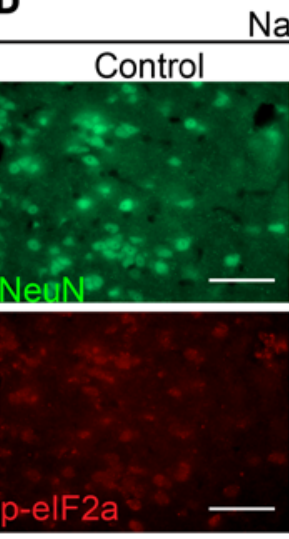

Naive

EAE
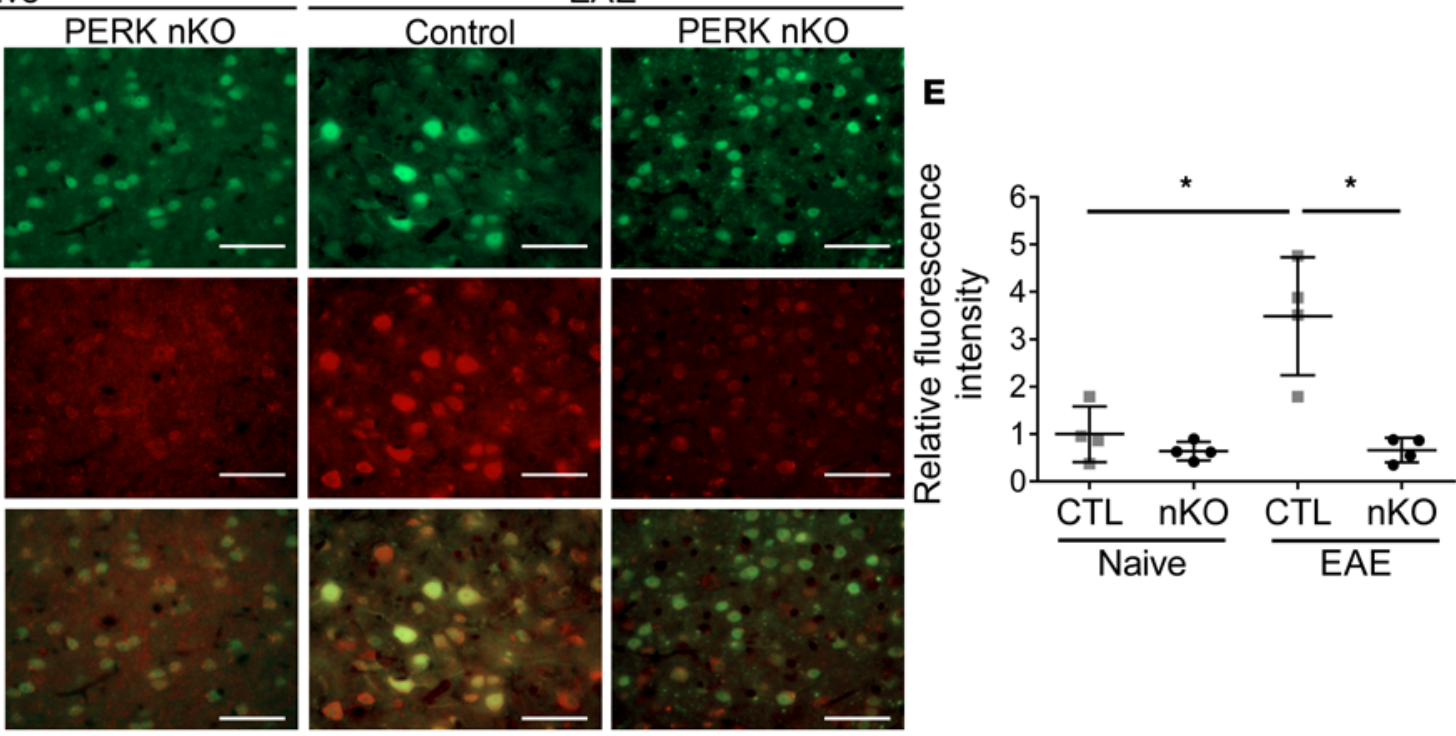

Figure 3. Neuron-specific PERK inactivation did not affect EAE initiation but impaired EAE resolution. (A) PERK-nKO mice with EAE exhibited persistent, severe neurological deficits, without signs of recovery. $n=12$ animals. Error bars represent SEM. Statistical analyses were done with a 2 -tailed Mann Whitney test, $* P<0.05$. (B and $\mathbf{C}$ ) Western blot analysis shows that the levels of ATF4 and CHOP were significantly increased in the brains of contro EAE mice at PID 22 compared with those in naive mice and that the levels of these proteins were significantly lower in the brains of PERK-nKO mice with EAE at PID 22. ( $D$ and E) NeuN and p-elF2 $\alpha$ double immunostaining shows that the level of p-elF2 $\alpha$ was markedly increased in neurons in the layer $V$ of the primary motor cortices of control EAE mice at PID 22 compared with that in naive mice. Importantly, the p-elF2 $\alpha$ level in cortical neurons was noticeably reduced in PERK-nKO mice with EAE compared with that in control EAE mice at PID 22. Scale bars: $50 \mu \mathrm{m}$. $n=4$ animals. Error bars represent SD. Statistical analyses were done with a 1-way ANOVA with a Tukey's post test, $* P<0.05$.

mice did not show signs of remission, displaying persistent, severe neurological deficits (Figure 3A). Western blot analysis showed that the levels of ATF4 and CHOP were significantly increased in the brains of control EAE mice compared with naive mice at the peak of disease, PID 22 (Figure 3, B and C). Importantly, the levels of ATF4 and CHOP were significantly decreased in the brains of PERK-nKO mice with EAE compared with those in control EAE mice at PID 22 (Figure 3, B and C). Moreover, NeuN and p-eIF2 $\alpha$ double immunostaining showed that the level of p-eIF $2 \alpha$ was significantly increased in neurons in the layer $\mathrm{V}$ of the primary motor cortices in control EAE mice compared with that in naive mice; however, the elevated level of p-eIF2 $\alpha$ was abrogated in PERK-nKO mice with EAE (Figure 3, D and E). Additionally, we found that 
the levels of p-eIF $2 \alpha$ and CHOP were significantly increased in neurons in the lumbar spinal cords of control EAE mice compared with those in naive mice at PID 22, but the elevated levels of p-eIF2 $\alpha$ and CHOP were abrogated in neurons of PERK-nKO mice with EAE (Supplemental Figure 1). These results demonstrate abrogation of the PERK-mediated ISR in neurons of PERK-nKO mice with EAE. Collectively, these data suggest that neuron-specific PERK inactivation does not affect EAE initiation but impairs EAE recovery.

There is evidence that axon degeneration is the root cause of permanent neurological disability in MS and EAE $(2,5,6)$. We assessed axon degeneration in the lumbar spinal cords of these EAE mice at the recovery stage, PID 38. In addition to Cre recombinase, Thy1/CreER $R^{T 2}$ mice also express enhanced yellow fluorescent protein (EYFP) under the control of the Thy1.2 promotor, which allows for the visualization of neurons and axons in the CNS using a fluorescent microscope (42). We used EYFP intensity to gauge axon loss in the lumbar spinal cord by quantifying the percentage of the white matter area that displayed noticeably reduced EYFP intensity. Interestingly, the percentage of the white matter area that displayed noticeably reduced EYFP intensity was dramatically increased in the lumbar spinal cords of PERK-nKO mice with EAE at PID 38 compared with that of control EAE mice (Figure 4, A-C). Accordingly, SMI-31 IHC showed that the number of SMI-31+ axons was dramatically decreased in the lumbar spinal cords of PERK-nKO mice with EAE at PID 38 compared with that of control EAE mice (Figure 4, D-F). Importantly, electron microscopy (EM) analysis verified that there were significantly fewer healthy-looking axons and more degenerating axons in the lumbar spinal cords of PERKnKO mice with EAE at PID 38 compared with those in control EAE mice (Figure 4, G-I). Thus, these results demonstrate that PERK inactivation in neurons facilitates axon degeneration during EAE.

We further determined oligodendrocyte loss, demyelination, and inflammation in the lumbar spinal cords of these EAE mice at PID 38. Immunostaining for aspartoacylase (ASPA), a marker for oligodendrocytes (30, 43), revealed a comparable number of oligodendrocytes in the lumbar spinal cords of PERK-nKO mice with EAE and control EAE mice at PID 38 (Figure 5, A-C). Double immunostaining for PDGFR $\alpha$ and Olig2, markers for oligodendrocyte precursor cells (OPCs), revealed a comparable number of OPCs that were positive for both PDGFR $\alpha$ and Olig2 in the lumbar spinal cords of PERK-nKO mice with EAE and control EAE mice at PID 38 (Figure 5, D-F). Conversely, myelin basic protein (MBP) IHC showed that the percentage of area in the white matter of the lumbar spinal cord that was demyelinated in PERK-nKO mice with EAE was significantly increased compared with that in control EAE mice at PID 38 (Figure 5, G-I). Toluidine blue staining also showed that the percentage of demyelinated area in the white matter of the lumbar spinal cords in PERKnKO mice with EAE was significantly increased compared with that in control EAE mice at PID 38 (Figure 5, J-L). Importantly, EM analysis confirmed that there were significantly fewer myelinated axons in the lumbar spinal cords of PERK-nKO mice with EAE compared with that in control EAE mice at PID 38 (Figure 4, G-I). Interestingly, $\mathrm{CD} 3$ immunostaining and $\mathrm{CD} 11 \mathrm{~b}$ immunostaining revealed significantly increased numbers of $\mathrm{CD}^{+} \mathrm{T}$ cells and $\mathrm{CD} 11 \mathrm{~b}^{+}$macrophages/microglia in the lumbar spinal cords of PERK-nKO mice with EAE compared with control EAE mice at PID 38 (Figure 5, M-R). Additionally, we determined oligodendrocyte loss and demyelination in the lumbar spinal cords of these EAE mice at the peak of disease (PID 22). We found that neuron-specific PERK inactivation did not alter the degree of oligodendrocyte loss, the number of OPCs, or the degree of demyelination in the lumbar spinal cord at the peak of disease (Supplemental Figure 2). Taken together, these data suggest that neuron-specific PERK inactivation facilitates axon degeneration in the CNS white matter during EAE and subsequently results in enhanced demyelination and inflammation.

Next, we determined the effects of PERK inactivation on neuron loss in the CNS gray matter of these EAE mice. One of our previous studies demonstrated significant but moderate neuron loss in the CNS gray matter in the MOG-EAE model, including the lumbar spinal cord, primary motor cortex, and cerebellum (39). On the other hand, it has been demonstrated that the efficiency of Cre-mediated recombination in Thy1/ $C r e E R^{T 2}$ mice is varied in different regions of the CNS, ranging from $35 \%$ to $97 \%$ (42). The recombination efficiency is high in the primary motor cortex (92\%), medium in the spinal cord (77\%), and low in the cerebellum (35\%). Thus, we assessed neuron loss in the layer $\mathrm{V}$ of the primary motor cortices in PERK-nKO mice with EAE and control EAE mice at PID 38. Quantitative NeuN IHC showed that the number of neurons in the layer $\mathrm{V}$ of the primary motor cortex was significantly reduced in PERK-nKO mice with EAE compared with that in control EAE mice at PID 38 (Figure 6, A-C). Nevertheless, CD3 immunostaining and CD11b immunostaining showed that there was no significant difference in the number of $\mathrm{CD}^{+} \mathrm{T}$ cells or $\mathrm{CD} 11 \mathrm{~b}^{+}$macrophages/microglia in the layer $\mathrm{V}$ of the primary motor cortices in PERK-nKO mice with EAE and control EAE mice at PID 38 (Figure 6, D-I). Collectively, these data suggest that neuron-specific PERK inactivation enhances neuron death but does not alter inflammation in the CNS gray matter during EAE. 
Control

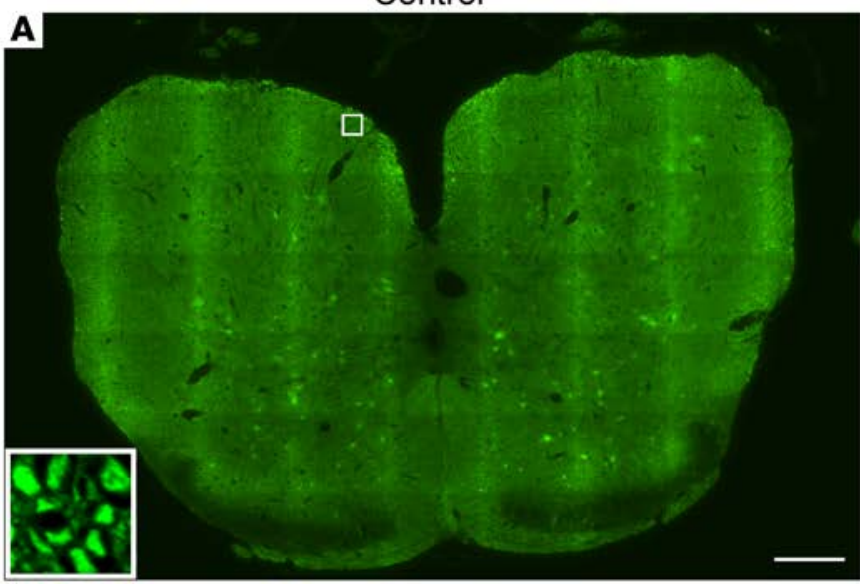

Control
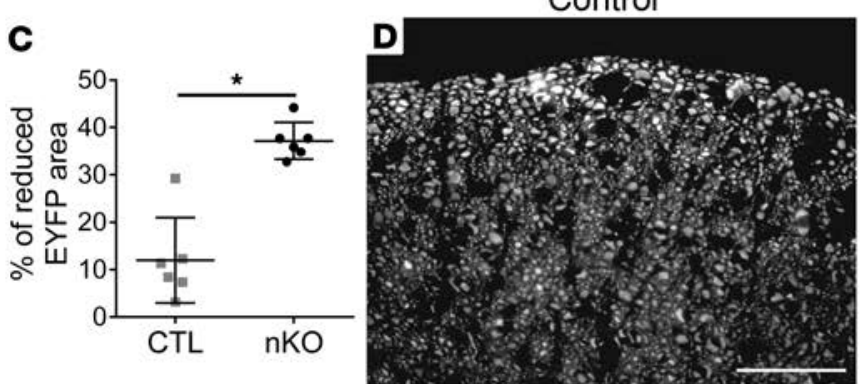
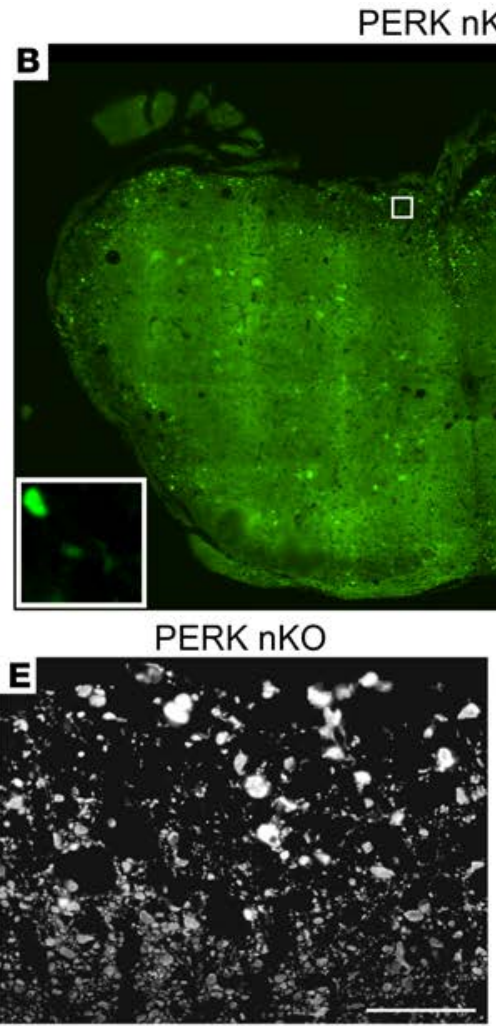

PERK nKO

$\square$
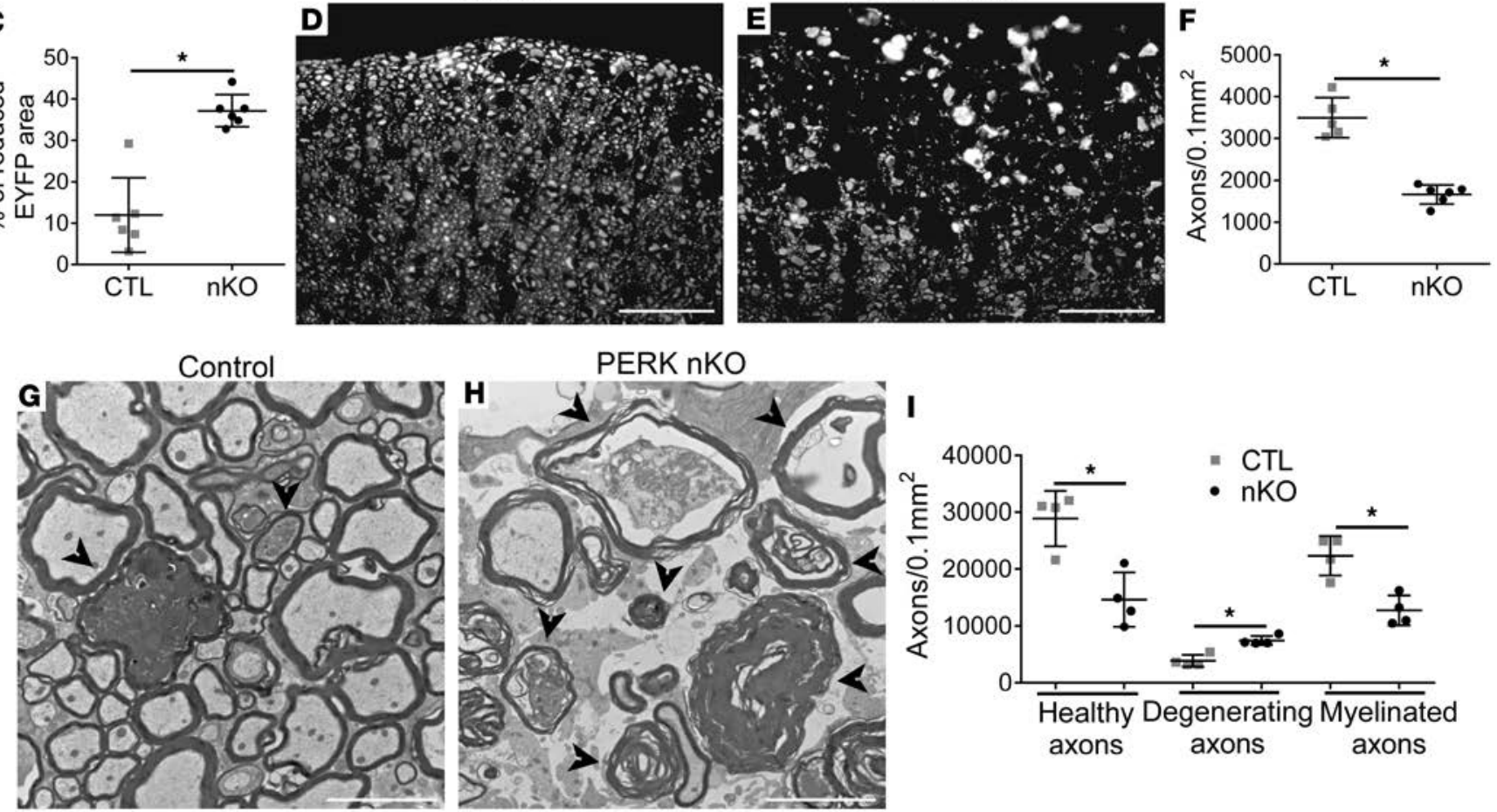

\section{I}

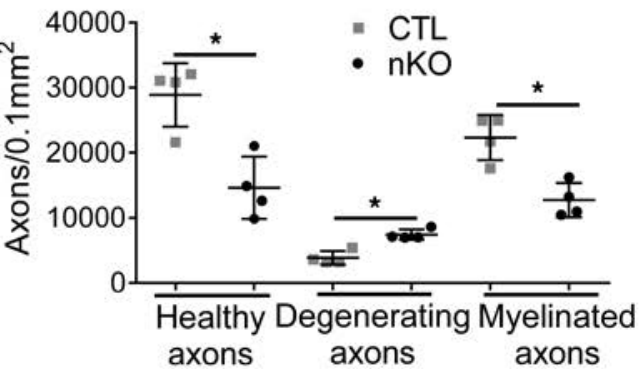

Figure 4. Neuron-specific PERK inactivation dramatically increased axon degeneration during EAE. (A-C) Fluorescent images of the whole lumbar spinal cord revealed that the percentage of the white matter area that displayed noticeably reduced EYFP intensity was significantly increased in PERK-nKO EAE mice compared with that in control EAE mice at PID 38. Insets show EYFP+ axons. $n=6$ animals. (D-F) SMI31 IHC revealed significantly fewer axons in the lumbar spinal cords of PERK-nKO mice with EAE compared with those in control EAE mice at PID 38. $n=6$ animals. (G-I) EM analysis confirmed that the number of degenerating axons (arrowheads) was significantly increased, and that the numbers of healthy-looking axons and myelinated axons were significantly decreased, in the lumbar spinal cords of PERK-nKO mice with EAE compared with those in control EAE mice at PID 38 . $n=4$ animals. Scale bars: $200 \mu \mathrm{m}$ (A and B); $50 \mu \mathrm{m}$ ( $\mathbf{D}$ and E); $5 \mu \mathrm{m}$ (G and H). Error bars represent SD. Statistical analyses were done with a 2 -tailed $t$ test, $* P<0.05$.

As described above, the Perk-KO allele was undetectable in the peripheral immune system or purified T cells of PERK-nKO mice (Figure 2A). To exclude the possibility that $\mathrm{T}$ cell priming was altered in PERK$\mathrm{nKO}$ mice with EAE, we performed in vitro $\mathrm{T}$ cell recall assays. Leukocytes were generated from the draining lymph nodes of PERK-nKO mice with EAE and control EAE mice at PID 9 and were restimulated with MOG 35-55 peptide. As expected, BrdU cell proliferation assay showed that neuron-specific PERK inactivation did not affect $\mathrm{T}$ cell proliferation in response to MOG 35-55 peptide (Figure 7A). Similarly, ELISA assays showed that neuron-specific PERK inactivation did not affect the ability of $\mathrm{T}$ cells to produce the cytokines IFN- $\gamma$, IL-17A, and IL-4 in response to MOG 35-55 peptide (Figure 7, B-D). Thus, these results demonstrate 

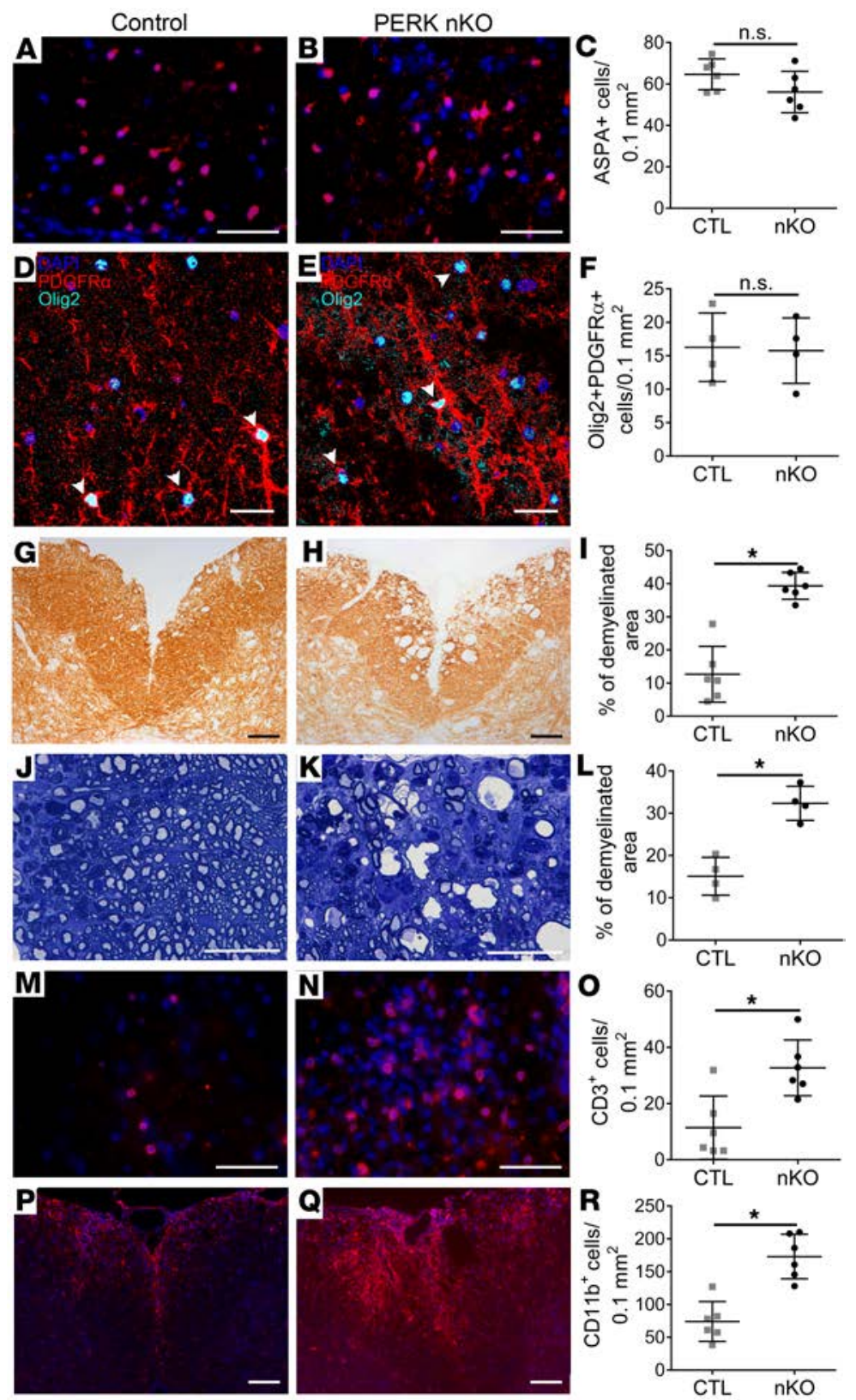

Figure 5. Neuron-specific PERK inactivation increased demyelination and inflammation in the lumbar spinal cords of $E A E$ mice. (A-C) ASPA immunostaining revealed that neuron-specific PERK inactivation did not significantly change oligodendrocyte numbers in the lumbar spinal cords of EAE mice at PID 38. $n=6$ animals. (D-F) PDGFR $\alpha$ and Olig2 double immunostaining revealed that neuron-specific PERK inactivation did not significantly change the number of OPCs that were positive for both PDCFR $\alpha$ and Olig2 (arrowheads) in the lumbar spinal cords of EAE mice at PID 38. $n=4$ animals. (G-I) MBP IHC revealed that PERK-nKO mice with EAE had a significantly increased demyelinated area in the lumbar spinal cord compared with that in control EAE mice at PID 38. $n=6$ animals. (J-L) Toluidine blue staining verified that PERK-nKO mice with EAE had a significantly increased demyelinated area in the lumbar spinal cord compared with that in control EAE mice at PID 38. $n=4$ animals. (M-0) CD3 immunostaining revealed that PERK-nKO mice with $E A E$ had a significantly increased number of T cells in the lumbar spinal cord compared with that in control EAE mice at PID 38. $n=6$ animals. (P-R) CD11b immunostaining revealed that PERK-nKO mice with EAE had a significantly increased number of macrophages/microglia in the lumbar spinal cord compared with that in control EAE mice at PID 38. $n=6$ animals. Scale bars: $50 \mu \mathrm{m}$ (A, B, M, and N); $20 \mu \mathrm{m}$ (D, E, J, and K); 100 $\mu \mathrm{m}(\mathbf{G}, \mathbf{H}, \mathbf{P}$, and $\mathbf{Q})$. Error bars represent SD. Statistical analyses were done with a 2-tailed $t$ test, ${ }^{*} P<0.05$.

that neuron-specific PERK inactivation does not alter T cell priming in the peripheral immune system during EAE. Taken together, our data demonstrate that PERK inactivation specifically in neurons exacerbates EAE-induced neurodegeneration, particularly axon degeneration, and subsequently results in persistent, severe neurological deficits in EAE mice, implying the neuroprotective effects of PERK activation in neurons in MS and EAE.

ATF4 was not required for neuron or axon survival in adult mice. ATF4 is the master transcription factor of the PERK-mediated ISR (17-19). We sought to determine the contribution of ATF4 to the neuroprotective effects of PERK activation in neurons during EAE. Global ATF4-KO mice display severe anemia and growth retardation and die prematurely $(44,45)$. Therefore, we first determined the effects of ATF4 inactivation on neurons and axons under physiological conditions using a mouse model that allowed for inactivation of ATF4 specifically in neurons. $A T F 4^{10 x P}$ mice that possess loxP sites flanking exons 2 and 3 of the Aft4 gene (46) were crossed with $T h y 1 / C r e E R^{T 2}$ mice, and the resulting progeny were further crossed with $A T F 4^{\text {lox } P}$ mice to obtain $A T F 4^{\text {loxP/loxP }}$; Thy $1 / C r e E R^{T 2}$ mice and $A T F 4^{\text {loxP/loxP }}$ mice. Nine-week-old $A T F 4^{\text {loxP/loxP}}$; Thy $1 / C r e E R^{T 2}$ mice were given i.p. injections of tamoxifen or vehicle daily for 8 consecutive days. The tamoxifen-treated mice did not exhibit any neurological phenotypes and were indistinguishable from the vehicle-treated mice. CNS tissues and other tissues were prepared from these mice 8 weeks after tamoxifen treatment. PCR analysis of genomic DNA revealed the deletion of exons 2 and 3 of the Atf4 gene selectively in the CNS of ATF4 ${ }^{\text {loxP } / \text { loxP }}$; Thy $1 / \mathrm{CreER}^{T 2}$ mice treated with tamoxifen (ATF4-nKO mice) but not in other organs of ATF4-nKO mice or in any organs of $A T F 4^{\text {loxP/loxP }}$; Thy $1 / \mathrm{CreER}^{T 2}$ mice treated with vehicle (control mice) (Figure 8A). H\&E staining showed that ATF4-nKO mice did not display any gross structural abnormalities in the CNS. Moreover, NeuN IHC revealed that ATF4-nKO mice had a comparable number of neurons in the layer V of the primary motor cortex compared with control mice (Figure 8, B-D). Similarly, SMI31 IHC revealed that the number of axons in the lumbar spinal cord was not compromised in ATF4-nKO mice compared with control mice (Figure 8, E-G). These data suggest that ATF4 is dispensable for neuron and axon survival in naive adult mice. 

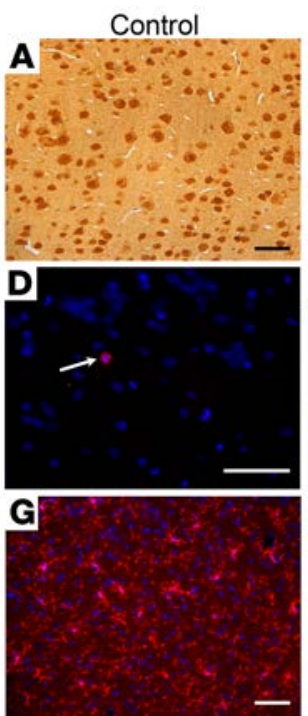
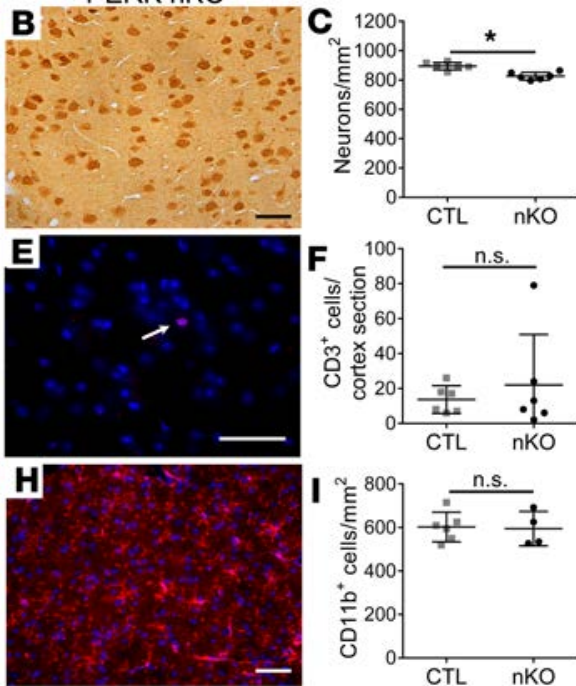
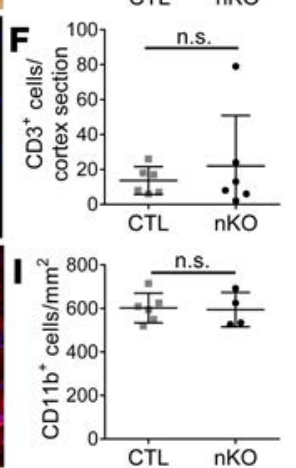

Figure 6. Neuron-specific PERK inactivation aggravated EAE-induced neuron loss in the CNS gray matter. (A-C) NeuN IHC revealed that PERK-nKO mice with EAE had significantly increased neuron loss in the layer $\mathrm{V}$ of the primary motor cortex compared with control EAE mice at PID 38. $n=6$ animals. (D-F) CD3 immunostaining revealed that neuron-specific PERK inactivation did not significantly change the number of $T$ cells (arrows) in the layer $V$ of the primary motor cortices of $E A E$ mice at PID 38. $n=6$ animals. (G-I) CD11b immunostaining revealed that neuron-specific PERK inactivation did not significantly change the number of macrophages/microglia in the layer $\mathrm{V}$ of the primary motor cortices of EAE mice at PID 38. $n=4-6$ animals. Scale bars: $50 \mu \mathrm{m}$. Error bars represent SD. Statistical analyses were done with a 2 -tailed $t$ test, $* P<0.05$

Neuron-specific ATF4 inactivation did not affect neurodegeneration in the CNS during EAE. To determine the effects of ATF4 inactivation in neurons in EAE, 9-week-old female $A T F 4^{\text {loxP } / l o x P}$; Thy $1 / C r e E R^{T 2}$ mice were given i.p. injections of tamoxifen daily for 8 consecutive days and then these mice were immunized with MOG 35-55 peptide to induce EAE at the age of 11 weeks (ATF4-nKO mice). For unknown reasons, the yield of $A T F 4^{\text {loxP/loxP }}$; Thy $1 / C r e E R^{T 2}$ mice was far below the Mendelian ratio. Thus, control EAE mice were agematched female $A T F 4^{10 x P / l o x P}$ mice treated with tamoxifen. Surprisingly, the disease course displayed by ATF4nKO mice with EAE was comparable to that of control EAE mice (Figure 9A). Western blot analysis showed that the levels of ATF4 and CHOP were significantly increased in the brains of control EAE mice compared with those in naive mice at the peak of disease, PID 24 (Figure 9, B and C). Importantly, the levels of ATF4 and CHOP were significantly decreased in the brains of ATF4-nKO mice with EAE compared with those of control EAE mice at PID 24 (Figure 9, B and C). Moreover, NeuN and p-eIF2 $\alpha$ double immunostaining showed that the level of p-eIF2 $\alpha$ was significantly increased in neurons in the layer $\mathrm{V}$ of the primary motor cortex in control EAE mice compared with naive mice at PID 24 and that the level of p-eIF2 $\alpha$ was not significantly altered in ATF4-nKO EAE mice compared with control EAE mice (Figure 9, D and E). Collectively, these data suggest that neuron-specific ATF4 inactivation does not influence EAE disease severity.

We further determined the effects of ATF4 inactivation specifically in neurons on axon loss, myelin damage, neuron loss, and inflammation in the CNS. Intriguingly, SMI31 IHC revealed a comparable number of axons in the lumbar spinal cords of ATF4-nKO mice with EAE and control EAE mice at PID 60 (Figure 10, A-C). MBP IHC showed that the percentage of area in the white matter of the lumbar spinal cord that was demyelinated in ATF4-nKO mice with EAE was comparable to that in control EAE mice at PID 60 (Figure 10, D-F). CD3 immunostaining and CD11b immunostaining revealed no significant change in the number of $\mathrm{CD}^{+} \mathrm{T}$ cells or $\mathrm{CD} 11 \mathrm{~b}^{+}$macrophages/microglia in the lumbar spinal cords of ATF4-nKO mice with EAE and control EAE mice at PID 60 (Supplemental Figure 3). Moreover, NeuN IHC revealed a comparable number of neurons in the layer V of the primary motor cortices in ATF4-nKO mice with EAE and control EAE mice at PID 60 (Figure 10, G-I). Similarly, CD3 immunostaining and $\mathrm{CD} 11 \mathrm{~b}$ immunostaining revealed no significant change in the number of $\mathrm{CD}^{+} \mathrm{T}$ cells or $\mathrm{CD} 11 \mathrm{~b}^{+}$macrophages/microglia in the layer V of the primary motor cortices in ATF4-nKO mice with EAE and control EAE mice at PID 60 (Supplemental Figure 4). Taken together, these data demonstrate that neuron-specific ATF4 inactivation does not influence axon loss, neuron loss, demyelination, or inflammation in the CNS of EAE mice, suggesting a minimal role of ATF4 in the viability of axons and neurons in MS and EAE.

\section{Discussion}

MS has traditionally been viewed as an autoimmune demyelinating disease of the CNS; however, it is becoming increasingly apparent that neurodegeneration is a major player in this disease $(1,2)$. Although MS is initiated by autoimmune attacks against oligodendrocytes and myelin, it is neurodegeneration, par- 
A
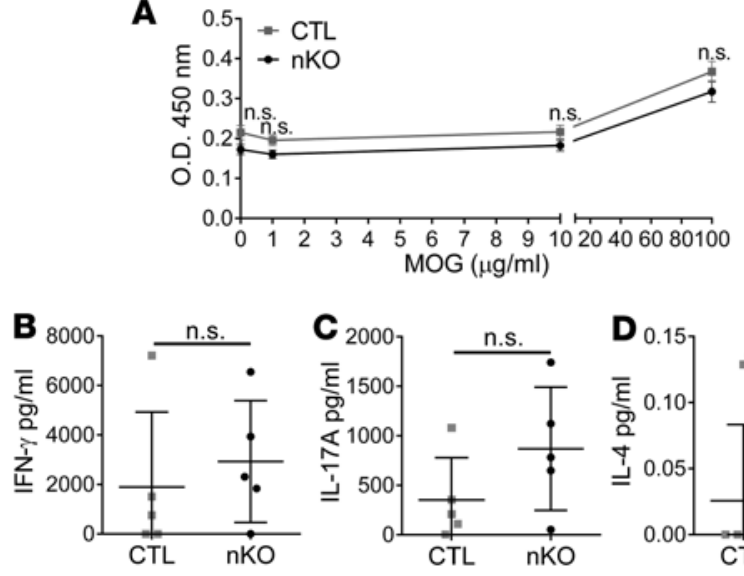

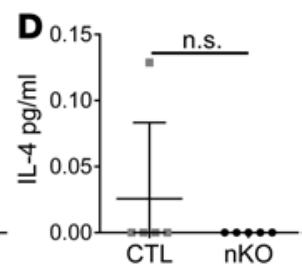

Figure 7. Neuron-specific PERK inactivation did not influence $T$ cell priming in the peripheral immune system during EAE. (A) BrdU cell proliferation assay shows that neuron-specific PERK inactivation did not significantly change $T$ cell proliferation in response to MOC 35-55 peptide. $n=5$ animals. Error bars represent SD. Statistical analyses were done with a 2-way ANOVA with a Tukey's post test. (B-D) ELISA assays show that neuron-specific PERK inactivation did not significantly change the ability of T cells to produce IFN- $\gamma$, IL-17A, and IL- 4 in response to MOC 35-55 peptide. $n=5$ animals. Error bars represent SD. Statistical analyses were done with a 2 -tailed $t$ test.

ticularly axon degeneration, which contributes the most to the progressive disability of MS patients (2-4). A number of studies have shown that the PERK-mediated ISR influences, positively or negatively, the viability of axons and neurons in various neurodegenerative diseases (33-35). Moreover, it is known that ER stress is an important player in regulating the viability and functions of inflammatory cells and resident cells in inflammatory lesions in various inflammatory diseases (22-24). Not surprisingly, there is evidence that the UPR is activated in neurons in MS patients $(26,36)$. Herein, we also demonstrated that the UPR, including the ATF6 $\alpha$ branch and the PERK-mediated ISR, was activated in neurons in the MOG-EAE model. Nevertheless, to date, attempts to assess the contribution of PERK activation in neurons to neurodegeneration and disease progression in MS and EAE have been unsuccessful.

To dissect the precise role of PERK activation in neurons during EAE, we generated mice that allow for controllable inactivation of PERK specifically in neurons. While a case study showed that a PERK mutation leads to early signs of neurodegeneration in a child (40), we found that PERK inactivation specifically in neurons did not affect neuron viability or axon integrity in adult mice. Our result suggests a minimal role of PERK in neurons under physiological conditions, which is consistent with previous studies using neuron-specific PERK conditional KO mice (47). Since global PERK deficiency in both human patients and animal models causes severe, complex phenotypes, such as pancreatic dysfunction, growth retardation, and premature death $(41,48,49)$, it is possible that early signs of neurodegeneration observed in the child carrying a PERK mutation are caused by non-cell autonomous effects of PERK deficiency on neurons. Interestingly, we found that neuron-specific PERK inactivation did not significantly alter EAE initiation but impaired clinical recovery from EAE. We also found that neuron-specific PERK inactivation significantly, although moderately, enhanced neuron loss in the CNS gray matter during EAE. Importantly, we found that neuron-specific PERK inactivation dramatically increased axon degeneration in the lumbar spinal cords of EAE mice. It is believed that axon degeneration is the leading cause of permanent disability in MS patients and a major contributor to EAE clinical symptoms $(2,5,6)$. The data in the present report suggest that neuron-specific PERK inactivation exacerbates axon degeneration, resulting in persistent, severe neurological deficits in mice undergoing EAE. Collectively, our results imply the neuroprotective effects of PERK activation in neurons in MS and EAE.

EAE is initiated by $\mathrm{T}$ cell priming by myelin components in the peripheral immune system, following which the myelin-reactive T cells enter the CNS where they induce inflammation that leads to tissue damage, including demyelination, oligodendrocyte death, axon degeneration, and neuron death $(4,13)$. Although evidence suggests that the PERK-mediated ISR influences the activity of inflammatory cells $(22,24)$, we showed here that the Perk-KO allele was undetectable in the peripheral immune system and purified $\mathrm{T}$ cells of PERK-nKO mice. We also verified that $\mathrm{T}$ cell priming was not altered in the peripheral immune system of PERK-nKO mice with EAE compared with control EAE mice. Moreover, we found that neuron-specific PERK inactivation moderately enhanced neuron loss but did not affect inflammation in the CNS gray matter during EAE. These data suggest that neuron-specific PERK inactivation does not influence inflammation during EAE through its direct actions in inflammatory cells. Nevertheless, we found that there was elevated inflammation in the lumbar spinal cords of PERK-nKO mice with EAE, 


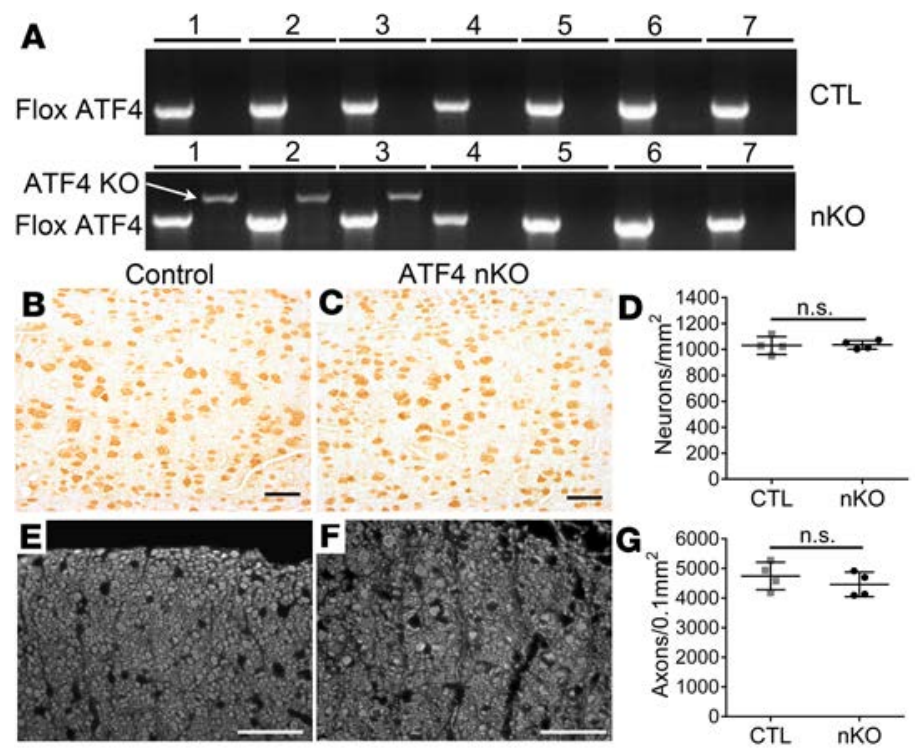

Figure 8. Neuron-specific ATF4 inactivation did not alter the viability of neurons or axons under physiological conditions. (A) PCR analysis using genomic DNA shows that the floxed Atf4 allele was present in all tissues in ATF4-nKO mice (nKO) or control mice (CTL) but the Atf4-KO (KO) allele was only present in the cerebral cortex, cerebellum, and spinal cord of ATF4-nKO mice. Numbering above lanes is as follows: 1 , cerebral cortex; 2, cerebellum; 3, spinal cord; 4, optic nerve; 5, sciatic nerve; 6 , spleen; 7, lymph node. (B-D) NeuN IHC shows that neuron-specific ATF4 inactivation did not significantly change the number of neurons in layer $V$ of the primary motor cortices of adult mice. (E-C) SMI31 IHC shows that neuron-specific ATF4 inactivation did not significantly change the number of axons in the lumbar spinal cord of adult mice. Scale bars: 50 $\mu \mathrm{m} . n=4$ animals. Error bars represent SD. Statistical analyses were done with a 2-tailed $t$ test.

which was associated with dramatically increased axon degeneration and demyelination at the recovery stage of disease (PID 38). Tissue damage is known to promote inflammation (50). Several studies have demonstrated that enhanced tissue damage in the CNS during EAE facilitates inflammation (51-53). Thus, there is a possibility that elevated inflammation in the lumbar spine cords of PERK-nKO mice with EAE is elicited by increased axon degeneration and demyelination.

Oligodendrocytes are myelin-producing cells in the CNS and the primary target of autoimmune attacks in MS and EAE $(4,13)$. Conversely, oligodendrocytes can regenerate and repair myelin damage in MS and EAE lesions. A number of studies have shown that demyelination and remyelination occur simultaneously in the CNS of EAE mice $(31,54)$. During the acute stage of EAE, oligodendrocyte death and demyelination are predominant in the CNS of EAE mice. Oligodendrocyte regeneration and remyelination initiate during the acute stage of EAE and become robust at the recovery stage of EAE. We found that the degree of demyelination, the degree of oligodendrocyte loss, and the number of OPCs were comparable in the lumbar spinal cords of PERKnKO mice and control mice at the peak of EAE (PID 22). This result suggests that neuron-specific PERK inactivation does not affect oligodendrocyte death or demyelination at the acute stage of EAE. While we found increased axon degeneration, demyelination, and inflammation in the lumbar spinal cords of PERK-nKO mice compared with control mice at the recovery stage of EAE (PID 38), the numbers of oligodendrocytes and OPCs were comparable in these two groups of mice at PID 38. This result likely reflects that neuron-specific PERK inactivation does not influence oligodendroglia regeneration at the recovery stage of EAE. It is well documented that healthy axons are required for myelin generation, maintenance, and regeneration under normal and disease conditions (2-6). Thus, these data also support the notion that enhanced myelin damage in PERK-nKO mice with EAE at the recovery stage of EAE results from increased axon degeneration.

ATF4, the master transcription factor of the PERK-mediated ISR, is responsible for inducing many cytoprotective genes that preserve cell viability and function under stressful conditions (17-19). A number of studies have shown that ATF4 activation influences, positively or negatively, the viability of neurons and axons in various neurodegenerative diseases (55-57). As expected, we demonstrated that ATF4 was activated in neurons during EAE and that ATF4 activation was abrogated in neurons of PERK-nKO mice with EAE. We further tested whether the neuroprotective effects of PERK activation in neurons in MS and EAE are mediated by ATF4. We generated mice that allow for controllable inactivation of ATF4 specifically in neurons and found that ATF4 was dispensable for neuron and axon survival under physiological conditions. Surprisingly, we found that neuron-specific ATF4 inactivation did not alter EAE clinical symptoms and had no effect on axon loss, neuron loss, demyelination, and inflammation in the CNS of EAE mice. This finding suggests that ATF4 is not a major player in regulating the viability of axons and neurons in MS and EAE. In parallel to this result, a previous study has shown that global deletion of CHOP does not alter EAE disease severity (58). Taken together, these data rule out the possibility that ATF4 is involved in the neuroprotective effects of PERK activation in neurons in MS and EAE. 
A
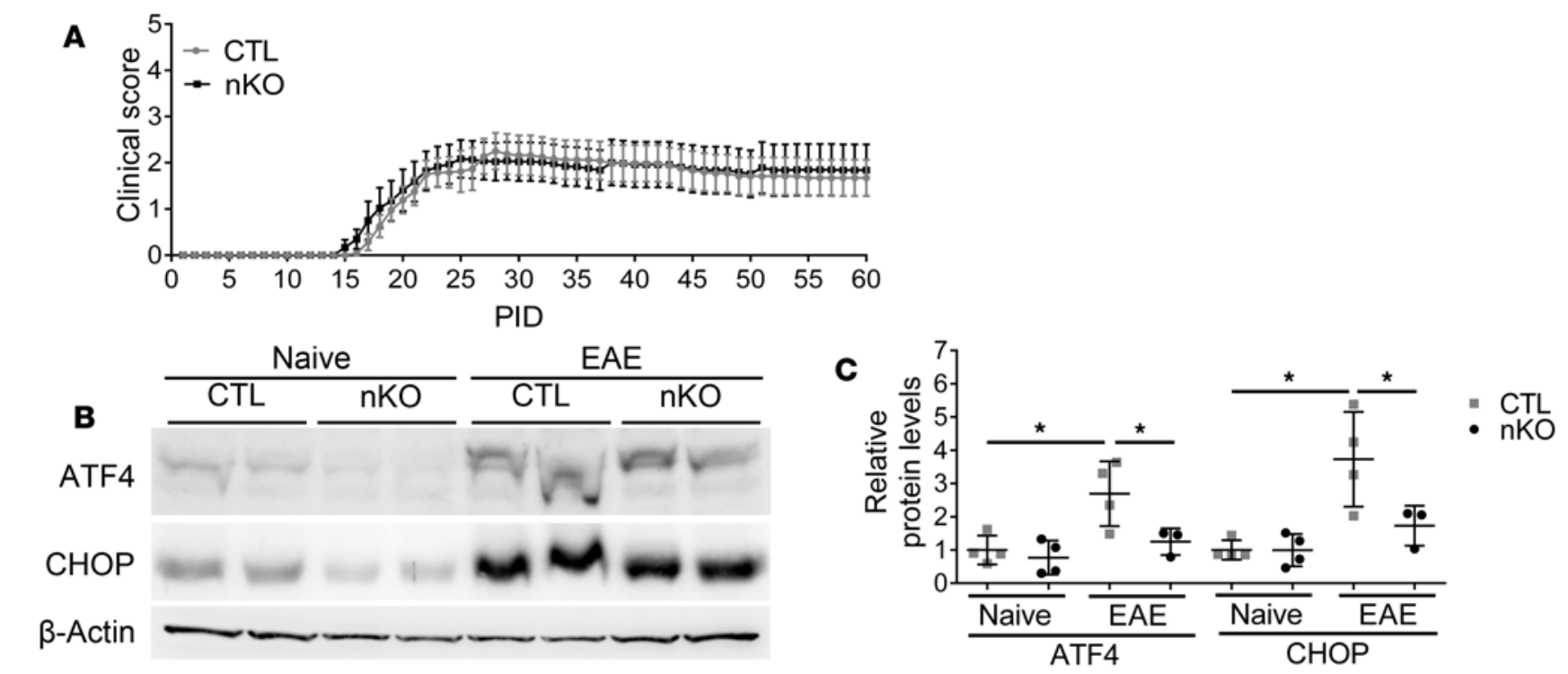

$\underline{D}$

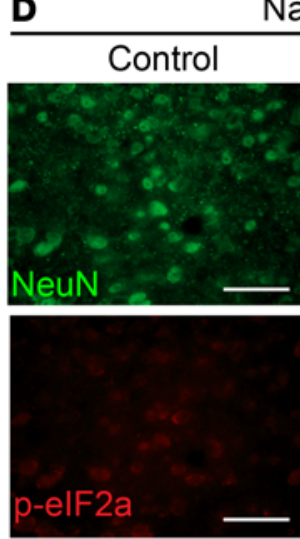

Naive ATF4 nKO
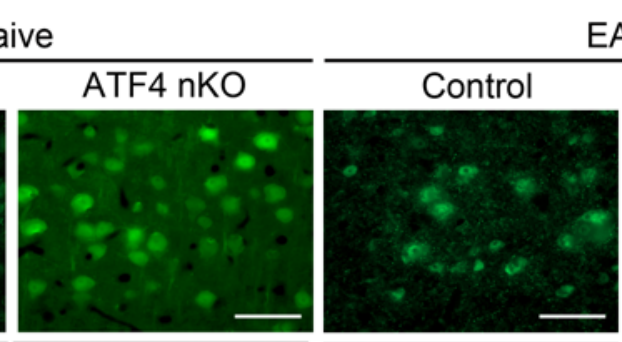

EAE
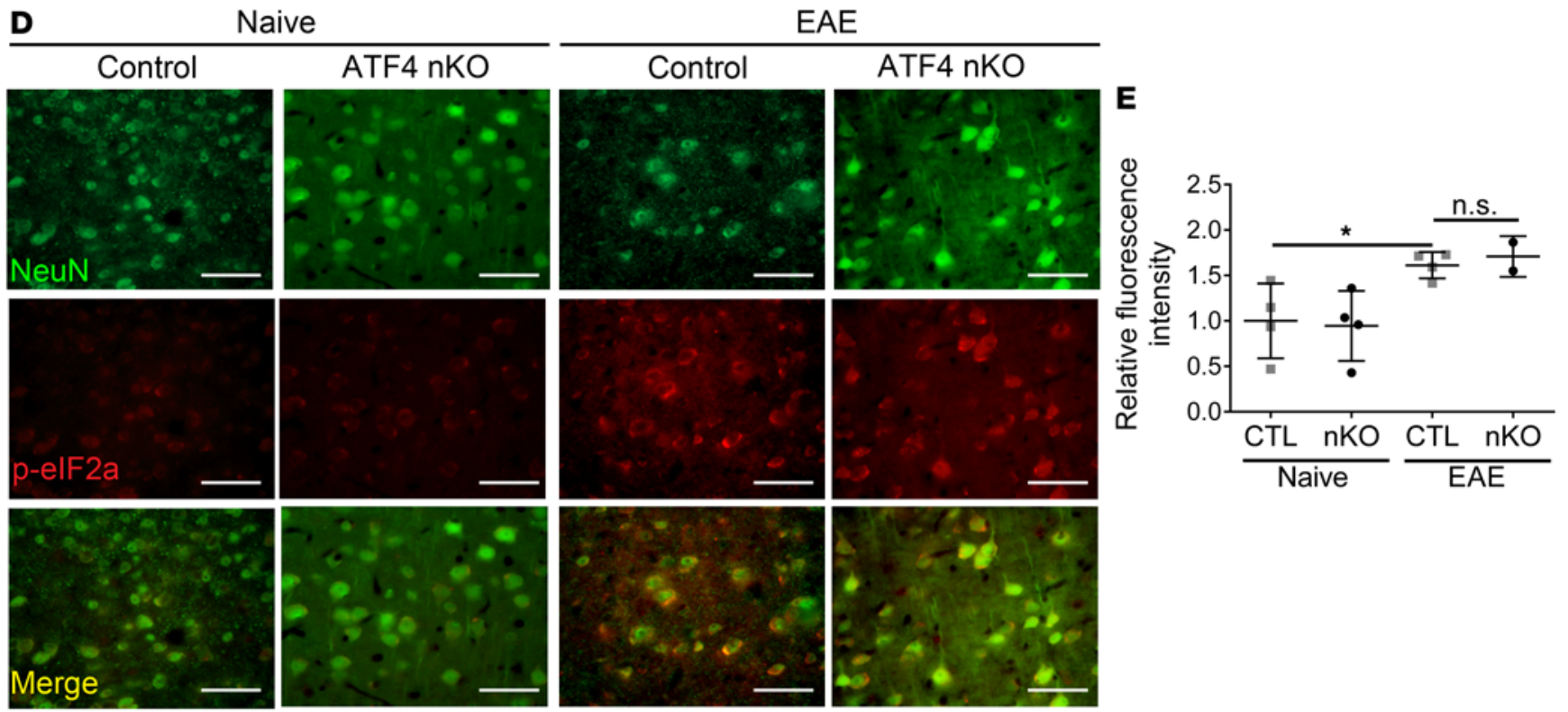

Figure 9. Neuron-specific ATF4 inactivation did not alter EAE disease course. (A) Neuron-specific ATF4 inactivation did not significantly alter the clinical course of EAE. $n=13$ animals. Error bars represent SEM. Statistical analyses were done with a 2-tailed Mann Whitney test. (B and C) Western blot analysis shows that the levels of ATF4 and CHOP were significantly increased in the brains of control EAE mice at PID 24 compared with those in naive mice and that the levels of these proteins were significantly decreased in the brains of ATF4-nKO mice with EAE compared with those in control EAE mice. $n=3-4$ animals. ( $D$ and $\mathbf{E}$ ) NeuN and p-elF2 $\alpha$ double immunostaining shows that the level of p-elF2 $\alpha$ was markedly increased in neurons in the layer $V$ of the primary motor cortices of control EAE mice at PID 24 compared with that in naive mice. Importantly, the p-elF2 $\alpha$ level in cortical neurons was comparable in ATF4-nKO mice with EAE and control EAE mice at PID 24. $n=2-4$ animals. Scale bars: $50 \mu \mathrm{m}$. Error bars represent SD. Statistical analyses were done with a 1-way ANOVA with a Tukey's post test, ${ }^{*} P<0.05$.

While ATF4 is generally considered to be the major transcription factor activated by PERK, PERK is also known to activate the transcription factor NF- $\kappa B$ by inhibiting the translation of its repressor, I $\mathrm{B} \alpha$, through phosphorylation of $\operatorname{eIF} 2 \alpha(30,59,60)$. A number of studies have suggested the neuroprotective effects of NF- $\mathrm{KB}$ activation in neurodegenerative diseases $(61,62)$. Importantly, a previous study showed that blockage of NF- $\mathrm{KB}$ activation specifically in neurons impairs EAE disease recovery and enhances EAE-induced axon degeneration and demyelination in the lumbar spinal cord (63), findings which are highly similar to the clinical and pathological features displayed by PERK-nKO mice with EAE. Intriguingly, the active form of $\mathrm{NF}-\mathrm{\kappa B}$ p65 immunostaining showed that the number of $\mathrm{EYFP}^{+}$neurons that were positive for the active form of $\mathrm{p} 65$ was significantly increased in the layer $\mathrm{V}$ of the primary motor cortices of control EAE mice as compared with that in naive mice at PID 22 and that the number of $\mathrm{EYFP}^{+}$neu- 


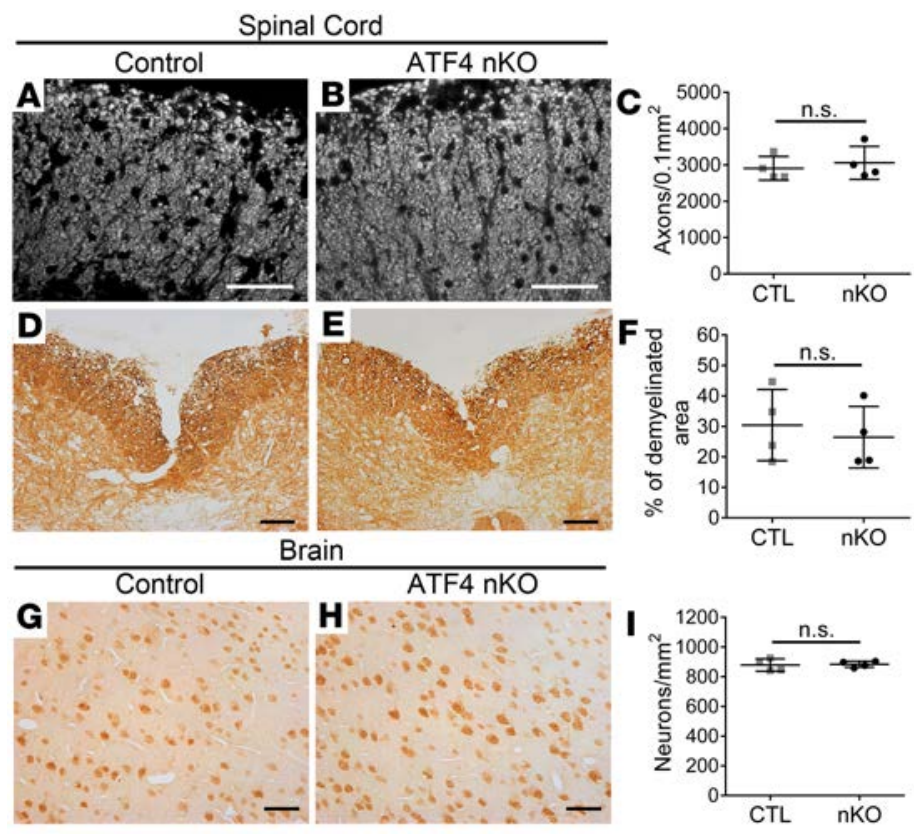

Figure 10. Neuron-specific ATF4 did not affect axon loss, demyelination, or neuron loss during EAE. (A-C) SMI31 IHC shows that neuron-specific ATF4 inactivation did not change the number of axons in the lumbar spinal cords of EAE mice at PID 60. (D-F) MBP IHC shows that neuron-specific ATF4 inactivation did not change the extent of demyelination in the lumbar spinal cords of EAE mice at PID 60. (G-I) NeuN IHC shows that neuron-specific ATF4 inactivation did not significantly change the number of neurons in the layer $V$ of the primary motor cortex at PID 60. Scale bars: $50 \mu \mathrm{m}$ (A, B, G, and H); $100 \mu \mathrm{m}$ (D and $\mathbf{F}$ ). $n=4$ animals. Error bars represent SD. Statistical analyses were done with a 2-tailed $t$ test.

rons that were positive for the active form of p65 was significantly decreased in PERK-nKO mice with EAE as compared with that in control EAE mice (Supplemental Figure 5). Collectively, these data show that the detrimental effects of PERK inactivation in neurons during EAE are accompanied by decreased NF- $\mathrm{KB}$ activation in neurons and raise the possibility that PERK activation in neurons prevents axon degeneration and neuron death in MS and EAE by activating NF- $\mathrm{BB}$.

In summary, we showed here that neuron-specific PERK inactivation impaired EAE resolution and exacerbated EAE-induced axon degeneration and neuron loss; however, neuron-specific ATF4 inactivation did not influence EAE clinical symptoms or EAE-induced neurodegeneration. These findings imply the neuroprotective effects of PERK activation in neurons in MS and EAE via ATF4-independent mechanisms. As such, this work may provide a foundation for designing new treatments that delay disability progression in MS patients through attenuation of axon degeneration and neuron loss by enhancing the activity of PERK in neurons.

\section{Methods}

Mice, tamoxifen treatment, and EAE immunization. Wild-type mice, PERK ${ }^{\text {loxP }}$ mice (41) (The Jackson Laboratory, stock 023066), $A T F 4^{\text {loxP }}$ mice (46), and Thy1/CreER ${ }^{T 2}$ mice (42) (The Jackson Laboratory, stock 012708) were on the C57BL/6J background. PERK ${ }^{\text {loxP }}$ mice were crossed with Thy1/CreER ${ }^{T 2}$ mice, and the resulting progeny were further crossed with $P E R K^{\text {loxP }}$ mice to obtain $P E R K^{\text {loxP/loxP }}$; Thy1/CreER $R^{T 2}$ mice, $P E R K^{\text {loxP/loxP }}$ mice, and Thy $1 / C r e E R^{T 2}$ mice. $A T F 4^{\text {loxP }}$ mice were crossed with Thy1/CreER $R^{T 2}$ mice, and the resulting progeny were further crossed with $A T F 4^{10 x P}$ mice to obtain $A T F 4^{\text {loxP } / \text { loxP }}$; Thy $1 / C r e E R^{T 2}$ mice and $A T F 4^{\text {loxP/loxP }}$ mice. Genotypes were determined by PCR from DNA extracted from tail tips, as described in previous papers $(41,42,46)$. To induce CreER ${ }^{\mathrm{T} 2}$-mediated recombination, 7-week-old PERK ${ }^{\text {loxP/loxP }}$; Thy $1 / \mathrm{CreER} \mathrm{R}^{\mathrm{T2}}$ mice and 9-week-old ATF4 ${ }^{\text {loxP/loxP}}$; Thy 1/CreER $R^{T 2}$ mice were given i.p. injections of tamoxifen or vehicle daily for 8 consecutive days, as described in a previous paper (42). To assess $\mathrm{CreER}^{\mathrm{T} 2}$-mediated recombination in the CNS of these mice, genomic DNA was isolated from the indicated tissues and PCR was performed as described in previous papers $(41,46)$.

To induce EAE, adult female mice were injected subcutaneously in the flank/tail base with $200 \mu \mathrm{g}$ MOG 35-55 peptide emulsified in complete Freund's adjuvant (BD Biosciences) supplemented with 600 $\mu \mathrm{g}$ Mycobacterium tuberculosis (strain H37Ra; BD Biosciences). Two i.p. injections of 400 ng pertussis toxin (List Biological Laboratories) were given 0 and 48 hours later. Clinical scores (0, healthy; 1, flaccid tail; 2 , ataxia and/or paresis of hind limbs; 3, paralysis of hind limbs and/or paresis of forelimbs; 4, tetraparalysis; 5 , moribund or death) were recorded daily $(29,30,37,38)$.

IHC. Anesthetized mice were perfused through the left cardiac ventricle with $4 \%$ paraformaldehyde in PBS. Half sagittal brain and the cephalic half of the lumbar spinal cord (L1-L3) (39) were post fixed in $4 \%$ paraformaldehyde for 2 hours, cryoprotected in 30\% sucrose for 48 hours, embedded in optimum cutting 
temperature compound, and frozen on dry ice. Frozen sections were cut using a cryostat at a thickness of $10 \mu \mathrm{m}$. The other half sagittal brain and the caudal half of the lumbar spinal cord (L3-L5) (39) were post fixed in 4\% paraformaldehyde for 72 hours, dehydrated through graded alcohols, and embedded in paraffin wax. Paraffin sections were cut using a microtome at a thickness of $5 \mu \mathrm{m}$. Fluorescein (1:100, Vector Laboratories, anti-mouse, catalog FI-2000), Cy3 (1:500, MilliporeSigma, anti-rat, catalog AP136C; anti-mouse, catalog AP124C; anti-rabbit, catalog AP132C), Alexa Fluor 647 (1:500, Thermo Fisher Scientific, catalog A21245), or enzyme-labeled secondary antibodies (1:200, Vector Laboratories, anti-mouse, catalog BA-2000) were used for detection. Immunohistochemical detection of MBP (1:1000; Sternberger Monoclonals, catalog SMI-99P-100), CD3 (1:50; Santa Cruz Biotechnology, catalog sc-18843), CD11b (1:50; MilliporeSigma, catalog CBL1313), SMI31 (1:1000, Covance, catalog SMI-31R-100), NeuN (1:100, MilliporeSigma, catalog MAB337), CHOP (rabbit 1:100, provided by David Ron at University of Cambridge, Cambridge, United Kingdom), p-eIF2 $\alpha$ (1:100, Cell Signaling Technology, catalog 9721), PDGFR $\alpha$ (1:100, MilliporeSigma, cata$\log$ CBL1366), Olig2 (1:300, MilliporeSigma, catalog AB9610), the active form of p65 (1:50; MilliporeSigma, catalog MAB3026), and ASPA (1:1000, provided by M.A. Aryan Namboodiri at Uniformed Services University of the Health Sciences, Bethesda, Maryland, USA) were performed. Fluorescent-stained sections were mounted with Vectashield mounting medium with DAPI (Vector Laboratories) and visualized with a Zeiss Axioskop 2 fluorescence microscope or an Olympus FV1000 confocal microscope.

For quantification of cells and axons in the lumbar spinal cords, we counted immunopositive cells or axons in an area of $0.1 \mathrm{~mm}^{2}$ within the anterior funiculus medially next to the anterior median fissure in the lumbar spinal cord as described in our previous paper $(29,30,38,39)$. For quantitative MBP IHC analysis, we calculated the percentage of the demyelinated area in the lumbar spinal cord by normalizing the demyelinated area against the total white matter area. The total white matter area and the demyelinated area in the lumbar spinal cord were measured with the NIH ImageJ software (http://rsb.info.nih.gov/ ij/; RRID:SCR_003070) as described in our previous paper $(29,30,38)$. To quantify the percentage of the white matter area in the lumbar spinal cord that displayed noticeably reduced EYFP intensity, EYFP fluorescence was visualized with a Nikon TiE inverted fluorescence microscope. We measured the total white matter area and the area that displayed noticeably reduced EYFP intensity with the NIH ImageJ software and then normalized the area with noticeably reduced EYFP intensity against the total white matter area. For quantification of neurons in the layer $\mathrm{V}$ of the primary motor cortex, 5 - $\mu \mathrm{m}$ thick serial sections were cut from Bregma lateral $1.08 \mathrm{~mm}$ to $1.32 \mathrm{~mm}$, every 10 th section was stained with NeuN, and immunopositive cells were counted as described in our previous paper (39).

The intensity of p-eIF $2 \alpha$ and CHOP fluorescence in neurons was measured as described previously $(38,64)$. Briefly, the NIH ImageJ software was used to measure the mean fluorescence of individual neurons as well as several readings of background fluorescent levels. The total corrected cellular fluorescence (TCCF) was determined using the following formula: TCCF = integrated density - (area of selected cell $\times$ mean fluorescence of background readings). p-eIF2 $\alpha$ and CHOP TCCF levels were normalized to the level of expression in the control mice.

Toluidine blue staining and EM analysis. Mice were deeply anesthetized and perfused with PBS containing $4 \%$ paraformaldehyde and $2.5 \%$ glutaraldehyde. The lumbar spinal cord was removed, processed, and embedded in resin as described previously (28). For toluidine blue staining, 1- $\mu \mathrm{m}$ sections were cut, stained with toluidine blue, and analyzed as previously described $(29,30,38)$. The demyelinated area and total white matter area in the lumbar spinal cord were measured using the NIH ImageJ software, and the percentage of the demyelinated area was calculated by normalizing the demyelinated area against the total white matter area. For EM analysis, ultrathin sections of the lumbar spinal cord were cut, stained with uranyl acetate and lead citrate, and analyzed as described previously $(30,31)$. We counted the number of healthy-looking axons, the number of degenerating axons, and the number of myelinated axons.

Western blot analysis. Brains were harvested from mice, rinsed in ice-cold PBS, and homogenized with a motorized homogenizer as previously described $(30,38)$. Extracts were incubated on ice for 15 minutes and cleared by centrifugation at 18,800 $\mathrm{g}$ for 30 minutes twice. The protein concentration was determined using the DC Protein Assay (Bio-Rad Laboratories). The extracts $(50 \mu \mathrm{g})$ were separated by on SDS-PAGE gels and transferred to nitrocellulose membranes. The membranes were incubated with a primary antibody against CHOP (1:1000, Santa Cruz Biotechnology, catalog sc-56107), ATF4 (1:1000, Abcam, catalog ab50546), ATF6 $\alpha$ (1:500; Cosmo Bio Co, catalog BAM-73-505-EX), or actin (1:5000, MilliporeSigma, catalog A2103), overnight, followed by an HRP-conjugated secondary antibody (1:1000, Vector Laboratories, 
anti-mouse, catalog PI-2000; anti-rabbit, PI-1000). The chemiluminescent signal was detected using the ECL Detection Reagents (GE Healthcare Biosciences). The intensity of the chemiluminescence signals of CHOP, ATF4, and actin were quantified using the NIH ImageJ software.

Real-time PCR. The cortices and hippocampi were isolated from the brains of mice. RNA was isolated from the tissue using Trizol reagent (Invitrogen) and treated with DNaseI (Invitrogen) to eliminate genomic DNA. Reverse transcription was performed using the iScript cDNA Synthesis Kit (Bio-Rad Laboratories). TaqMan real-time PCR for GAPDH and PERK was performed with LightCycler 480 Probes Master (Roche Diagnostics Corporation) on the LightCycler 480 System (Roche) as described in one of our previous papers (65).

$T$ cell proliferation assay and cytokine assays. Draining lymph nodes (inguinal, brachial, and axillary) were isolated from EAE mice at PID 9, homogenized into a single-cell suspension, and plated in 96-well microtiter plates in triplicate. The cells were incubated with MOG $35-55$ peptide $(0,1,10$, or $100 \mu \mathrm{g} / \mathrm{ml})$ at $37^{\circ} \mathrm{C}$ and $5 \% \mathrm{CO}_{2}$. $20 \mu \mathrm{BrdU}$ labeling solution (MilliporeSigma) was added to the culture media after 48 hours and incubated for a further 24 hours. Cell proliferation was determined using the Colorimetric BrdU Cell Proliferation kit (MilliporeSigma) according to manufacturer's instructions. Cytokines in the culture supernatants were quantified using the ELISA kits (IFN- $\gamma$, IL-4, IL-17A, BioLegend) according to the manufacturer's instructions.

$T$ cell purification. Spleens were isolated from mice and homogenized, and red blood cells were lysed with ACK lysis buffer (Thermo Fisher Scientific). Cells were labeled with CD45 and CD3, and CD45 CD3 ${ }^{+}$ T cells were isolated using a FACSAria II cell sorter (BD Biosciences).

Statistics. All statistical analyses were performed using GraphPad Prism 6 (GraphPad Software, RRID:SCR_002798). EAE clinical score data are presented as mean \pm SEM and were evaluated using a 2-tailed Mann Whitney test to compare clinical scores daily. All other data are expressed as mean \pm SD. Comparisons between two groups were statistically evaluated by a 2-tailed $t$ test. Multiple comparisons were statistically evaluated by a 1-way ANOVA with a Tukey's post test or a 2-way ANOVA with a Tukey's post test. $P$ values of less than 0.05 were considered significant.

Study approval. All animal procedures were conducted in complete compliance with the NIH Guide for the Care and Use of Laboratory Animals (National Academies Press, 2011) and were approved by the Institutional Animal Care and Use Committee of the University of Minnesota.

\section{Author contributions}

WL and SS designed the experiments, performed the experiments, analyzed the data, and wrote the manuscript. YY designed the experiments, performed the experiments, and analyzed the data. MS performed the experiments and analyzed the data. SW performed the experiments. GK provided $A T F 4^{10 x P}$ mice.

\section{Acknowledgments}

We thank M.A. Aryan Namboodiri for providing the antibody against ASPA. We thank David Ron for providing the polyclonal CHOP antibody. WL is supported by grants from the NIH (NS094151 and NS105689) and the National Multiple Sclerosis Society (RG 5239-A-3).

Address correspondence to: Wensheng Lin, Institute for Translational Neuroscience, University of Minnesota, 2101 6th Street SE, WMBB4-140, Minneapolis, MN 55455, USA. Phone: 612.626.5079; Email: linw@umn.edu.

1. Frohman EM, Racke MK, Raine CS. Multiple sclerosis--the plaque and its pathogenesis. N Engl J Med. 2006;354(9):942-955.

2. Reich DS, Lucchinetti CF, Calabresi PA. Multiple sclerosis. N Engl J Med. 2018;378(2):169-180.

3. Mahad DH, Trapp BD, Lassmann H. Pathological mechanisms in progressive multiple sclerosis. Lancet Neurol. 2015;14(2):183-193.

4. Baecher-Allan C, Kaskow BJ, Weiner HL. Multiple Sclerosis: mechanisms and Immunotherapy. Neuron. 2018;97(4):742-768.

5. Trapp BD, Peterson J, Ransohoff RM, Rudick R, Mörk S, Bö L. Axonal transection in the lesions of multiple sclerosis. $N$ Engl J Med. 1998;338(5):278-285.

6. Trapp BD, Nave KA. Multiple sclerosis: an immune or neurodegenerative disorder? Annu Rev Neurosci. 2008;31:247-269.

7. Papadopoulos D, Dukes S, Patel R, Nicholas R, Vora A, Reynolds R. Substantial archaeocortical atrophy and neuronal loss in multiple sclerosis. Brain Pathol. 2009;19(2):238-253.

8. Haider L, et al. The topograpy of demyelination and neurodegeneration in the multiple sclerosis brain. Brain. 2016;139(Pt 3):807-815.

9. De Stefano N, et al. Assessing brain atrophy rates in a large population of untreated multiple sclerosis subtypes. Neurology. 2010;74(23):1868-1876.

10. Vigeveno RM, Wiebenga OT, Wattjes MP, Geurts JJ, Barkhof F. Shifting imaging targets in multiple sclerosis: from inflamma- 
tion to neurodegeneration. J Magn Reson Imaging. 2012;36(1):1-19.

11. Steinman L. Assessment of animal models for MS and demyelinating disease in the design of rational therapy. Neuron. 1999;24(3):511-514

12. Lassmann H. Axonal and neuronal pathology in multiple sclerosis: what have we learnt from animal models. Exp Neurol. 2010;225(1):2-8.

13. Lassmann H, Bradl M. Multiple sclerosis: experimental models and reality. Acta Neuropathol. 2017;133(2):223-244.

14. Glass CK, Saijo K, Winner B, Marchetto MC, Gage FH. Mechanisms underlying inflammation in neurodegeneration. Cell. 2010;140(6):918-934.

15. Siffrin V, Vogt J, Radbruch H, Nitsch R, Zipp F. Multiple sclerosis - candidate mechanisms underlying CNS atrophy. Trends Neurosci. 2010;33(4):202-210.

16. Larochelle C, Uphaus T, Prat A, Zipp F. Secondary progression in multiple sclerosis: neuronal exhaustion or distinct pathology? Trends Neurosci. 2016;39(5):325-339.

17. Marciniak SJ, Ron D. Endoplasmic reticulum stress signaling in disease. Physiol Rev. 2006;86(4):1133-1149.

18. Walter P, Ron D. The unfolded protein response: from stress pathway to homeostatic regulation. Science. 2011;334(6059):1081-1086.

19. Pakos-Zebrucka K, Koryga I, Mnich K, Ljujic M, Samali A, Gorman AM. The integrated stress response. EMBO Rep. 2016;17(10):1374-1395.

20. Tabas I, Ron D. Integrating the mechanisms of apoptosis induced by endoplasmic reticulum stress. Nat Cell Biol. 2011;13(3):184-190.

21. Hetz C, Papa FR. The unfolded protein response and cell fate control. Mol Cell. 2018;69(2):169-181.

22. Zhang K, Kaufman RJ. From endoplasmic-reticulum stress to the inflammatory response. Nature. 2008;454(7203):455-462.

23. Lin W, Popko B. Endoplasmic reticulum stress in disorders of myelinating cells. Nat Neurosci. 2009;12(4):379-385

24. Grootjans J, Kaser A, Kaufman RJ, Blumberg RS. The unfolded protein response in immunity and inflammation. Nat Rev Immunol. 2016;16(8):469-484.

25. Mháille AN, et al. Increased expression of endoplasmic reticulum stress-related signaling pathway molecules in multiple sclerosis lesions. J Neuropathol Exp Neurol. 2008;67(3):200-211.

26. Ní Fhlathartaigh M, et al. Calreticulin and other components of endoplasmic reticulum stress in rat and human inflammatory demyelination. Acta Neuropathol Commun. 2013;1:37.

27. Way SW, Popko B. Harnessing the integrated stress response for the treatment of multiple sclerosis. Lancet Neurol. 2016;15(4):434-443.

28. Lin W, Kemper A, Dupree JL, Harding HP, Ron D, Popko B. Interferon-gamma inhibits central nervous system remyelination through a process modulated by endoplasmic reticulum stress. Brain. 2006;129(Pt 5):1306-1318.

29. Lin W, et al. The integrated stress response prevents demyelination by protecting oligodendrocytes against immune-mediated damage. J Clin Invest. 2007;117(2):448-456.

30. Lin W, et al. Oligodendrocyte-specific activation of PERK signaling protects mice against experimental autoimmune encephalomyelitis. J Neurosci. 2013;33(14):5980-5991.

31. Lin Y, et al. PERK activation preserves the viability and function of remyelinating oligodendrocytes in immune-mediated demyelinating diseases. Am J Pathol. 2014;184(2):507-519.

32. Hussien Y, Cavener DR, Popko B. Genetic inactivation of PERK signaling in mouse oligodendrocytes: normal developmental myelination with increased susceptibility to inflammatory demyelination. Glia. 2014;62(5):680-691.

33. Li S, Yang L, Selzer ME, Hu Y. Neuronal endoplasmic reticulum stress in axon injury and neurodegeneration. Ann Neurol. 2013;74(6):768-777.

34. Scheper W, Hoozemans JJ. The unfolded protein response in neurodegenerative diseases: a neuropathological perspective. Acta Neuropathol. 2015;130(3):315-331.

35. Hetz C, Saxena S. ER stress and the unfolded protein response in neurodegeneration. Nat Rev Neurol. 2017;13(8):477-491.

36. Stone S, Lin W. The unfolded protein response in multiple sclerosis. Front Neurosci. 2015;9:264.

37. Stone S, Jamison S, Yue Y, Durose W, Schmidt-Ullrich R, Lin W. NF- $\kappa$ B activation protects oligodendrocytes against inflammation. J Neurosci. 2017;37(38):9332-9344.

38. Stone S, Wu S, Jamison S, Durose W, Pallais JP, Lin W. Activating transcription factor $6 \alpha$ deficiency exacerbates oligodendrocyte death and myelin damage in immune-mediated demyelinating diseases. Glia. 2018;66(7):1331-1345.

39. Stanojlovic M, Pang X, Lin Y, Stone S, Cvetanovic M, Lin W. Inhibition of vascular endothelial growth factor receptor 2 exacerbates loss of lower motor neurons and axons during experimental autoimmune encephalomyelitis. PLOS ONE. 2016;11(7):e0160158.

40. Bruch J, Kurz C, Vasiljevic A, Nicolino M, Arzberger T, Höglinger GU. Early neurodegeneration in the brain of a child without functional PKR-like endoplasmic reticulum kinase. J Neuropathol Exp Neurol. 2015;74(8):850-857.

41. Zhang P, et al. The PERK eukaryotic initiation factor 2 alpha kinase is required for the development of the skeletal system, postnatal growth, and the function and viability of the pancreas. Mol Cell Biol. 2002;22(11):3864-3874.

42. Heimer-McGinn V, Young P. Efficient inducible Pan-neuronal cre-mediated recombination in SLICK-H transgenic mice. Genesis. 2011;49(12):942-949.

43. Madhavarao CN, Moffett JR, Moore RA, Viola RE, Namboodiri MA, Jacobowitz DM. Immunohistochemical localization of aspartoacylase in the rat central nervous system. J Comp Neurol. 2004;472(3):318-329.

44. Masuoka HC, Townes TM. Targeted disruption of the activating transcription factor 4 gene results in severe fetal anemia in mice. Blood. 2002;99(3):736-745

45. Yang X, et al. ATF4 is a substrate of RSK2 and an essential regulator of osteoblast biology; implication for Coffin-Lowry Syndrome. Cell. 2004;117(3):387-398.

46. Yoshizawa T, et al. The transcription factor ATF4 regulates glucose metabolism in mice through its expression in osteoblasts. $J$ Clin Invest. 2009;119(9):2807-2817.

47. Ma T, et al. Suppression of eIF2 $\alpha$ kinases alleviates Alzheimer's disease-related plasticity and memory deficits. Nat Neurosci. 2013;16(9):1299-1305 
48. Delépine M, Nicolino M, Barrett T, Golamaully M, Lathrop GM, Julier C. EIF2AK3, encoding translation initiation factor 2-alpha kinase 3, is mutated in patients with Wolcott-Rallison syndrome. Nat Genet. 2000;25(4):406-409.

49. Harding HP, et al. Diabetes mellitus and exocrine pancreatic dysfunction in perk-/- mice reveals a role for translational control in secretory cell survival. Mol Cell. 2001;7(6):1153-1163.

50. David S, Zarruk JG, Ghasemlou N. Inflammatory pathways in spinal cord injury. Int Rev Neurobiol. 2012;106:127-152.

51. Hisahara S, et al. Targeted expression of baculovirus p35 caspase inhibitor in oligodendrocytes protects mice against autoimmune-mediated demyelination. EMBO J. 2000;19(3):341-348.

52. Hisahara S, Yuan J, Momoi T, Okano H, Miura M. Caspase-11 mediates oligodendrocyte cell death and pathogenesis of autoimmune-mediated demyelination. J Exp Med. 2001;193(1):111-122.

53. Mc Guire C, et al. Oligodendrocyte-specific FADD deletion protects mice from autoimmune-mediated demyelination. $J$ Immunol. 2010;185(12):7646-7653.

54. Franklin RJ, Ffrench-Constant C. Remyelination in the CNS: from biology to therapy. Nat Rev Neurosci. 2008;9(11):839-855

55. Sun X, et al. ATF4 protects against neuronal death in cellular Parkinson's disease models by maintaining levels of parkin. $J$ Neurosci. 2013;33(6):2398-2407.

56. Baleriola J, et al. Axonally synthesized ATF4 transmits a neurodegenerative signal across brain regions. Cell. 2014;158(5):1159-1172

57. Pitale PM, Gorbatyuk O, Gorbatyuk M. Neurodegeneration: keeping ATF4 on a tight leash. Front Cell Neurosci. 2017;11:410.

58. Deslauriers AM, et al. Neuroinflammation and endoplasmic reticulum stress are coregulated by crocin to prevent demyelination and neurodegeneration. J Immunol. 2011;187(9):4788-4799.

59. Deng J, et al. Translational repression mediates activation of nuclear factor kappa B by phosphorylated translation initiation factor 2. Mol Cell Biol. 2004;24(23):10161-10168.

60. Lin Y, Jamison S, Lin W. Interferon- $\gamma$ activates nuclear factor- $\kappa$ B in oligodendrocytes through a process mediated by the unfolded protein response. PLoS One. 2012;7(5):e36408

61. Lanzillotta A, et al. NF-кB in Innate Neuroprotection and age-related neurodegenerative diseases. Front Neurol. 2015;6:98.

62. Yue Y, Stone S, Lin W. Role of nuclear factor $\kappa \mathrm{B}$ in multiple sclerosis and experimental autoimmune encephalomyelitis. Neural Regen Res. 2018;13(9):1507-1515.

63. Emmanouil M, et al. Neuronal I kappa B kinase beta protects mice from autoimmune encephalomyelitis by mediating neuroprotective and immunosuppressive effects in the central nervous system. J Immunol. 2009;183(12):7877-7889.

64. McCloy RA, Rogers S, Caldon CE, Lorca T, Castro A, Burgess A. Partial inhibition of Cdk1 in G 2 phase overrides the SAC and decouples mitotic events. Cell Cycle. 2014;13(9):1400-1412.

65. Ho Y, et al. PERK activation promotes medulloblastoma tumorigenesis by attenuating premalignant granule cell precursor apoptosis. Am J Pathol. 2016;186(7):1939-1951. 